\title{
A Representation Theory for Morphological Image and Signal Processing
}

\author{
PETROS MARAGOS, MEMBER, IEEE
}

\begin{abstract}
This paper presents a unifying theory for many concepts and operations encountered in or related to morphological image and signal analysis. This unification requires a set-theoretic methodology, where signals are modeled as sets, systems (signal transformations) are viewed as set mappings, and translation-invariant systems are uniquely characterized by special collections of input signals. This approach leads to a general representation theory, in which any translation-invariant, increasing, upper semicontinuous system can be represented exactly as a minimal nonlinear superposition of morphological erosions or dilations. In this representation, many similarities and a few differences are observed between systems processing binary or multilevel signals, and continuous-domain or discrete-domain signals. The theory is used to analyze some special cases of image/signal analysis systems, such as morphological filters, median and order-statistic filters, linear filters, and shape recognition transforms. Although the developed theory is algebraic, its prototype operations are well suitable for shape analysis; hence, the results of this study also apply to systems that extract information about the geometrical structure of signals.
\end{abstract}

Index Terms-Image/signal processing, mathematical morphology, nonlinear/linear filtering, shape analysis, systems representation.

\section{INTRODUCTION AND SUMmaRy}

$\mathrm{T}$ HIS paper develops a representation theory unifying many systems ("systems" are defined here as signalto-signal transformations) encountered in or related to morphological image and signal processing. By "morphological signal processing"' is meant in this paper not only the area of mathematical morphology [1], [2] as an image analysis method, but also the use of its implicit signal analysis techniques to study arbitrary signals and systems. Although the theory is motivated by specific existing systems related to morphology, it is very general and refers to all systems that share three properties: translation-invariant, increasing (preserve a signal ordering), and semicontinuous (insensitive to very fine signal details).

Many theoretical results concerning the operations of mathematical morphology can be found in [1]-[15], [46]. These operations have been applied successfully to a broad variety of image processing/analysis tasks (including noise suppression. image enhancement, coding, feature

Manuscript received June 19, 1987; revised April 11, 1988. This work is based on the author's Ph.D. thesis, Georgia Institute of Technology, Atlanta, July 1985, and was supported by the Joint Services Electronics Program under Grant DAAG-84-K-0024. The writing and refinements of this paper were done at Harvard University while the author was supported by the National Science Foundation under Grant CDR-85-00108.

The author is with the Division of Applied Sciences, Harvard University, Cambridge, MA 02138.

IEEE Log Number 8926696. extraction, thinning, texture analysis, and shape recognition) encountered in diverse areas such as biomedical image processing, cellular automata, electron microscopy, astronomy, and automated industrial visual inspection. 'The basic concepts and analytic tools behind morphological operations can be found for binary signals in set theory and integral geometry, and for multilevel signals in measure theory, convex analysis and fuzzy set theory. The ideas needed from these areas of mathematics are elementary, but can become effective tools for analysis of arbitrary signals and systems. For example the theoretical analysis of median and rank-order filters has been simplified and extended using such "morphological" techniques in [13]. In addition, the relationship among morphological filters, median-type filters, and even classical linear filters goes much deeper: they can all be represented in terms of a minimal nonlinear superposition of morphological erosions or dilations [11]-[13]. One goal then of this paper is to introduce a general representation theory which includes these fundamental results as special cases.

Currently, there are many commercial computer architectures for digital image analysis whose main operations are combinations of erosions and dilations. Early examples include [21]-[23]; for some recent examples see the session on morphological systems of the workshop [24]. In addition, there are VLSI, optical/electronic, and analog optical implementations of rank order filters (whose special cases are the simple erosions and dilations) or morphological filters [25]-[28]. Therefore, from an applications viewpoint, our representation theory establishes the capabilities and limitations of all these computer architectures and implementations by finding the general class of signal operations that they can perform. Further, the ever-increasing industrial need in automated visual systems calls for low-cost machine vision modules that can do a variety of complex image processing/analysis tasks based on a rather small set of available simple image operations. Hence, given the wide applicability of erosions/dilations, their parallellism, and their simple implementations, the work in this paper theoretically sup-

'Since we do not attempt here a survey of applications of mathematical morphology, a complete list of such references lies beyond the scope of this paper. A few examples can be found in [2], [5], [16]-[19], the session on morphology of the conference [20], and the tutorial parts of [10], [12], [14]. 
ports a general purpose vision (software or hardware) module that can perform erosions/dilations. Although several morphology-based algebraic systems have been proposed (e.g., see [2], [8], [9], [29]) for image processing, it is known from [30], [31] that erosions/dilations are insufficient by themselves to represent all possible image operations. Image algebras have been developed [30], [31]-[33], [34] which represent all digital image-to-image operations as a finite composition of a few basic operations, which include erosions/dilations. These image algebras contain as (morphological) subalgebras all the operations representable via erosions/dilations only. Ritter et al. [31] showed that a subalgebra of their full image algebra generalizes the theory of mathematical morphology.

From a theoretical viewpoint, this paper unifies the representation of previously totally unrelated systems, e.g., morphological filters used in image analysis and median (or other order-statistic based) filters used in robust statistics. It also provides a common mathematical framework and analytic tools for a large class of linear and nonlinear filters. Finally, a motivation for our research is our belief that it helps conceptually and offers insight to know that a particular system together with many others sharing a few common properties result from the combination of a few simple generic systems.

This paper, which reports work from [9], is organized as follows: in Section II we provide a set-theoretic methodology for signals and systems suitable for the representation theory; a brief review of the definitions of the required basic morphological filters; and some concepts related to systems obeying a "threshold" superposition that will help us bridge the gap between systems processing binary versus multilevel signals. In Section III-A we summarize Matheron's [1] results on using the kernel (a special collection of input signals) of translation-invariant set mappings to show that any translation-invariant increasing system processing binary signals is a union of erosions by its kernel sets, and also an intersection of dilations by the kernel sets of a dual system. Our first major contribution begins with Section III-A where we extend Matheron's work by introducing a kernel representation for systems processing multilevel signals and by showing that any such translation-invariant increasing system is the supremum of erosions by its kernel functions, and also the infimum of dilations by the kernel functions of a dual system. These kernel representations of systems require an infinite number of elements. Seeking to eliminate any redundancy in the kernel, we introduce in Section IV the concept of the basis as the collection of minimal (with respect to a signal ordering) kernel elements. The basis is an infinitely smaller subcollection of the kernel, and sometimes it is finite. Our major contributions in Section IV is to show that the basis of translation-invariant, increasing, upper semicontinuous systems processing multilevel (binary) signals exists, and such systems can be represented exactly as the supremum (union) of erosions by their basis functions (sets). These results are devel- oped for systems processing signals of both discrete and continuous domain. Although the latter case forces us to use a mathematical sophistication that could perhaps be avoided for discrete systems, we believe it is worthwhile for two reasons: 1) theoretical completeness, and 2) practical motivations arising from the existence of optical analog implementations [27], [28] that perform morphological filtering on images with continuous domain and range. Another contribution in Sections III-B and IV concerns the special class of systems that can process both multilevel and binary signals without altering this feature and commute with thresholding; the application of our theory to these systems yields their representation in terms only of the kemel of their corresponding binary-signalprocessing systems, which are easier to analyze and implement. Finally, in Section V we use the general theory from Sections III and IV to obtain new realizations for some special classes of systems, i.e., morphological filters, median and rank order filters, linear filters, and window-transforms for shape recognition. In Scctions V-B and V-C we also survey some results from [9], [12], [13]. Some of these results were obtained via combinatorial proofs which were unrelated to morphology and different for each class of systems. Here we rederive these results using the theory of this paper as a manifestation of the generality of the theory, from which the special cases result as simple corollaries.

The proofs of all the major theoretical results that are new contributions are contained in the Appendix.

\section{BACKGROUND}

\section{A. Set Representations of Signals}

Following the classification of [12] for $m$-dimensional ( $m$-D; $m=1,2,3, \cdots)$ signals, in our analysis we shall always use the distinction that an $m$-D function represents and refers to an $m-\mathrm{D}$ multilevel signal, whereas an $m$-D set represents an $m$-D binary (two-level) signal. This induces a similar classification for $m$-D systems into either function-processing (FP) systems, which can accept as inputs and produce as outputs $m$-D multilevel signals, or into set-processing (SP) systems, whose both inputs and outputs are $m$-D binary signals. A subclass of $m$-D FP systems can produce a binary $m$-D output signal whenever the input is also an $m$-D binary signal; these are called function- and set-processing (FSP) systems.

Notation: $\boldsymbol{R}$ and $\boldsymbol{Z}$ denote the set of real and integer numbers, respectively. $\boldsymbol{D}=\boldsymbol{R}^{m}$ or $\boldsymbol{Z}^{m}$ is the domain set on which signals are defined. $\boldsymbol{V}=\boldsymbol{R}$ or $\boldsymbol{Z}$ is the range set in which signals take values. Capital letters " $A, B$, $\cdots, X, Y^{\prime}$ denote sets; points of sets are denoted by lower case letters ' $a, b, \cdots, x, y, z$ '” ' $\varnothing, \in, \subseteq,(\cdot)^{c}$, $\cup, \cap, \times$ " denote, respectively, the empty set, set membership, inclusion, complementation, union, intersection, Cartesian product. $\{x: P\}$ denotes the set of points $x$ satisfying a property $P . f: A \rightarrow B$ denotes a mapping $f$ whose domain is $A$ and range is a subset of $B$. sup ( $\cdot)$, inf ( $\cdot$ ), $\max (\cdot)$, and $\min (\cdot)$ denote, respectively, the supre- 
mum, infimum, maximum, and minimum of sets in $\boldsymbol{R}$. Functions are denoted by " $f, g, h$ ". Capital (lowercase) Greek letters, e.g., $\Psi, \Phi(\psi, \phi)$ denote SP (FP) systems. If $f$ is input signal to the system $\psi, \psi(f)$ denotes the output signal. " $\Rightarrow, \Leftrightarrow, \forall$ " denote, respectively, "implies," "if and only if (iff)," and "for all."

It not otherwise stated, we henceforth work in the class ERV $(\boldsymbol{D})$ of all extended real-valued functions on $\boldsymbol{D}$, i.e., functions $f: D \rightarrow V \cup\{-\infty,+\infty\}$. The threshold sets (also called cross-sections in [2]) of such a function $f$ are the sets

$$
T_{a}(f) \stackrel{\text { def }}{=}\{x \in D: f(x) \geq a\}, \quad a \in \boldsymbol{V},
$$

which are generated by thresholding $f$ at any amplitude level $a$.

Theorem $I^{2}$ : a) The threshold sets $T_{a}(f), a \in V$, of an extended real-valued function $f(x), x \in D$, decrease as $a$ increases and obey a monotonic continuity; i.e., $\forall a, b \in$ V,

$$
\begin{aligned}
a<b & \Rightarrow T_{a} \supseteq T_{b}, \\
T_{b} & =\bigcap_{a<b} T_{a}(\text { if } \mathbf{V}=\mathbf{R}) .
\end{aligned}
$$

Further, $f$ can be reconstructed from its threshold sets:

$$
f(x)=\sup \left\{a \in V: x \in T_{a}(f)\right\}, \quad \forall x \in D .
$$

b) Let $\left\{G_{a} \subseteq D: a \in V\right\}$ be any set collection satisfying (2) (and (3) if $\boldsymbol{V}=\boldsymbol{R}$ ), and define a function $g(x)$ $=\sup \left\{a \in V: x \in G_{a}\right\}, x \in D$. Then, $T_{a}(g)=G_{a} \forall a \in$ V.

Reconstruction (4) leads us to allow our functions to take infinite values. That is, if for some $x_{0} \in D$ the set of $a$ 's in the brackets of (4) is equal to $\varnothing$ or $V$, then we simply set $f\left(x_{0}\right)=\sup (\varnothing)=-\infty$ or $f\left(x_{0}\right)=\sup (\boldsymbol{V})$ $=+\infty$. This formalism is very convenient for the nonlinear signal operations examined in this paper.

Another set representation of $f$ is its umbra [4], [5]

$$
U(f) \stackrel{\text { def }}{=}\{(x, a) \in D \times V: f(x) \geq a\},
$$

which uniquely characterizes $f$ since

$$
f(x)=\sup \{a \in V:(x, a) \in U(f)\}, \quad \forall x \in D .
$$

$U(f)$ is a subset of $\boldsymbol{E}=\boldsymbol{D} \times \boldsymbol{V}$. This definition of $\boldsymbol{E}$ implies that $\boldsymbol{E}=\boldsymbol{R}^{m+1}, \boldsymbol{R}^{m} \times \boldsymbol{Z}, \boldsymbol{Z}^{m} \times \boldsymbol{R}$, or $\boldsymbol{Z}^{m+1}$. The subsets of $\boldsymbol{E}$ in these four cases can represent, respectively, $m$-D signals whose choices for argument and amplitude span the four combinations of being continuous or discrete.

Set operations on threshold sets or umbrae induce certain nonlinear operations on functions. That is, let the functions $\left(\wedge_{i \in I} f_{i}\right)(x)=\inf _{i \in I}\left\{f_{i}(x)\right\}$ and $\left(\vee_{i \in I} f_{i}\right)(x)$ $=\sup _{i \in l}\left\{f_{i}(x)\right\}$ denote, respectively, the pointwise infimum and supremum of an indexed family $\left\{f_{i}: i \in I\right\}$ of functions. If the index set $I$ is finite, $\wedge(\vee)$ denotes the

\footnotetext{
${ }^{2}$ Theorem 1 is a generalized version of Serra's theorem $[2, p .426]$ that refers only to upper semicontinuous functions.
}

pointwise minimum (maximum). By using (1), (5), and the properties of sup/inf, the next results follow easily and establish that, intersection (union) of threshold sets or umbrae corresponds to $\wedge(\vee)$ of functions:

$$
\begin{gathered}
T_{a}(f)=\bigcap_{i \in I}^{\cup} T_{a}\left(f_{i}\right) \forall a \in V \Leftrightarrow U(f)=\bigcap_{i \in I}^{\cup} U\left(f_{i}\right) \\
T_{a}(f)=\bigcap_{i \in I} T_{a}\left(f_{i}\right) \forall a \in V \Leftrightarrow f=\bigwedge_{i \in I} f_{i} \\
T_{a}(f)=\bigcup_{i \in I} T_{a}\left(f_{i}\right) \forall a \in V \Rightarrow f=\bigvee_{i \in I} f_{i} \\
I \text { is finite } \Rightarrow T_{a}\left(\bigvee_{i \in I} f_{i}\right)=\bigcup_{i \in I} T_{a}\left(f_{i}\right) \forall a
\end{gathered}
$$

Such concepts are similar to the formalism encountered in the theory of fuzzy sets [36].

Finally, set inclusion between umbrae or threshold sets corresponds to an ordering relation between functions. That is, " $f$ precedes $g$ ", denoted as $f \leq g$, iff $f(x) \leq$ $g(x) \forall x \in D$; then

$$
f \leq g \Leftrightarrow U(f) \subseteq U(g) \Leftrightarrow T_{a}(f) \subseteq T_{a}(g) \forall a .
$$

\section{B. Morpohological Filters}

Morphological filters are nonlinear signal transformations that locally modify the geometrical fcatures of signals or image objects. Next we classify them into SP, FP, and FSP filters (systems).

$S P$ Filters [3], [1]: Consider sets $A, B$ in $E$. Let $A+b$ $=\{a+b: a \in A\}$ denote the (vector) translate of $A$ by $b \in \boldsymbol{E}$, and let $\breve{B}=\{-b: b \in B\}$ denote the reflection (symmetric) of $B$. The four basic SP morphological transformations of $A$ by $B$ are:

$$
\text { Dilation: } \begin{aligned}
A \oplus B & =\bigcup_{b \in B} A+b \\
& =\{z: A \cap(\check{B}+z) \neq \varnothing\},
\end{aligned}
$$

$$
\text { Erosion: } A \Theta B=\bigcap_{b \in B} A-b=\{z: B+z \subseteq A\} \text {, }
$$

Opening: $A \bigcirc B=(A \ominus B) \oplus B$,

Closing: $A \bigcirc B=(A \oplus B) \ominus B$.

The set dilation is the well-known Minkowski sum [3]. ${ }^{3}$

FP Filters [4], [5], [2], [12], [14]: As explained in [14] via the umbra homomorphism theorems, the dilation of a function $f$ by a function $g$ is defined indirectly by $U(f$

\footnotetext{
${ }^{3}$ In the recent literature on morphology, there are mainly two slightly different sets of definitions for (12)-(15): one of [1], [2] and another of [5], [14], which becomes identical if $B=\breve{B}$. Maragos and Schafer used in [9]-[13] the definitions from Matheron and Serra. In this paper we use Sternberg's definitions and the notation of Haralick $e t$ al. because they are simpler; our only difference is to use the group-theoretic notation $S+x$ for set translation. Note also that Hadwiger [3] originally called Minkowski sum and difference what we call here dilation and erosion.
} 
$\oplus g) \stackrel{\text { def }}{=} U(f) \oplus U(g)$. Then, if Spt $(f) \stackrel{\text { def }}{=}\{x \in D: f(x)$ $\neq-\infty\}$ denotes the support of $f$, the FP dilation of $f$ by $g$ is the function

$$
\begin{gathered}
(f \oplus g)(x)=\sup _{y \in[\operatorname{Spt}(f) \cap \operatorname{Spt}(\check{g})+x]}\{f(y)+g(x-y)\}, \\
x \in D,
\end{gathered}
$$

where $\breve{g}(x)=g(-x)$ is the reflection of $g$. The FP erosion of $f$ by $g$ is the function

$$
(f \ominus g)(x)=\inf _{y \in \operatorname{Spt}(g)+x}\{f(y)-g(y-x)\}
$$

if Spt $(g)+x \subseteq \operatorname{Spt}(f)$, and $-\infty$ if otherwise. The FP opening and closing of $f$ by $g$ are, respectively, the functions $f \bigcirc g=(f \ominus g) \oplus g$, and $f \bigcirc g=(f \oplus g) \ominus g$.

FSP Filters [6], [2]. As introduced in [2], if $\Phi(X)=$ $X \oplus B, X, B \subseteq D$, is an SP dilation filter and $f$ is an input function, the sets $\Phi\left[T_{a}(f)\right]$ satisfy the conditions to be the threshold sets of a function defined as the FSP dilation, $f \oplus B$, of $f$ by the set $B$; then (see Theorem 1)

$$
(f \oplus B)(x)=\sup \{f(x-y): y \in B\}, \quad x \in D .
$$

The FSP erosion of $f$ by $B$ is the function

$$
(f \ominus B)(x)=\inf \{f(x+y): y \in B\}, \quad x \in \boldsymbol{D} \text {. }
$$

The FSP opening and closing of $f$ by $B$ are, respectively, $f \bigcirc B=(f \ominus B) \oplus B$ and $f \bigcirc B=(f \oplus B) \ominus B$. The FSP erosion, dilation, opening, and closing of $f$ by $B$ result as simple cases from their FP counterparts whenever $g$ is binary, i.e., $g(x)=0$ for all $x \in \operatorname{Spt}(g)$. Then, if $B$ $=$ Spt $(g),(16)$ and (17) reduce to (18) and (19), respectively. For discrete-domain (e.g., sampled) signals and finite sets $B$, all the above FSP morphological filters become the moving local maximum/minimum filters introduced in [6].

\section{Threshold Superposition for FSP Systems}

Let $\phi$ be an FSP system, and let $\Phi$ be its corresponding SP system; i.e., if $\chi_{s}(z)$ is the characteristic function of a set $S\left(\chi_{s}(z)\right.$ is 1 if $z \in S$ and 0 if $\left.z \in S^{c}\right)$, then $\phi\left(\chi_{s}\right)=$ $\chi_{\phi(S)}$. We say that the $\phi$ obeys the threshold superposition property if

$$
[\phi(f)](x)=\sup \left\{a \in V: x \in \Phi\left[T_{a}(f)\right]\right\}
$$

for any function $f$. Hence, transforming $f$ by any FSP system $\phi$ satisfying (20) is equivalent to decomposing $f$ into its threshold sets $T_{a}(f)$, transforming each set $T_{a}(f)$ by the respective SP system $\Phi$, and then constructing the output function $\phi(f)$ from the transformed sets $\Phi\left[T_{a}(f)\right]$. FSP systems satisfying (20) are useful because the filtering of a multilevel signal by them reduces to filtering for binary signals, which is easier to analyze and implement.

A sufficient condition for threshold superposition is commuting with thresholding. That is, we say that an FSP system $\phi$ commutes with thresholding iff

$$
\Phi\left[T_{a}(f)\right]=T_{a}[\phi(f)], \quad \forall a \in V,
$$

for all $f$. Since (21) and (4) imply (20), an FSP system obeys the threshold superposition if it commutes with thresholding. Note that an FSP system satisfies (21) only if it satisfies some necessary conditions summarized in Lemma Al of the Appendix; a sufficient condition for (21) is provided in [12]. Examples of systems that commute with thresholding include the FSP erosion, dilation, opening, and closing as shown in [6], [2]. In addition, any cascade or parallel (using $\vee$ or $\wedge$ ) combination of FSP systems commuting with thresholding commutes with thresholding too. Similar threshold superpositions have been used extensively in the analysis of median and related nonlinear digital filters [37], [38], [13].

\section{Kernel Representations}

\section{A. SP Systems}

Consider the power set $\mathcal{P}(\boldsymbol{E})$ (class of all subsets of $\boldsymbol{E})$ and let $\delta \subseteq \rho(\boldsymbol{E})$ be henceforth a class of input sets (binary signals) closed under translation; i.e., $A+z \in S$, $\forall A \in S, \forall z \in E$. We say that an SP system $\Psi: \mathcal{S} \rightarrow \mathcal{P}(\boldsymbol{E})$ is translation-invariant (TI) iff $\Psi(A+z)=\Psi(A)+z$ $\forall A \in S, \forall z \in E$. TI systems have the exceedingly desirable property that the result of an image processing operation is independent of the exact location of the image object. The kernel of the TI system $\Psi$ is defined [1] as the following collection of input sets:

$$
\mathfrak{K}(\Psi) \stackrel{\text { def }}{=}\{A \in S: \overrightarrow{0} \in \Psi(A)\},
$$

where $\overrightarrow{0}$ denotes the zero vector of $\boldsymbol{E}$ (or of $\boldsymbol{D}$ according to the context). For example, the kernel of the SP erosion system $\Psi(A)=A \ominus B, A \in \mathcal{S}$, by a given $B \subseteq E$, is ${ }^{4}$ [see (22) and (13)]

$$
\mathcal{E}(B) \stackrel{\text { def }}{=} \mathcal{K}(A \mapsto A \ominus B)=\{A \in S: A \supseteq B\}
$$

Similarly, from (22) and (12), the kernel of the SP dilation $\Phi(A)=A \oplus B$ is

$$
\mathfrak{D}(B) \stackrel{\text { def }}{=} \mathfrak{K}(A \mapsto A \oplus B)=\{A \in \mathcal{S}: A \cap \breve{B} \neq \varnothing\} .
$$

A fundamental kemel property is the fact that from $\mathcal{K}(\Psi)$ we can uniquely reconstruct $\Psi$, because $\Psi(A)=$ $\{z \in \boldsymbol{E}: A-z \in \mathfrak{K}(\Psi)\}$.

Assume henceforth that we deal only with SP TI systems $\Psi$ that are nondegenerate: i.e., $\Psi(\varnothing)=\varnothing, \Psi(E)$ $=E$, and $\mathscr{K}(\Psi)$ contains more elements than the single set $\boldsymbol{E}$. Morcover, if $\left\{\Psi_{i}: i \in I\right\}$ is an indexed family of TI SP systems defined on $\mathcal{S}$, let us define their union and intersection superposition, respectively, as the systems $\left[\cup_{i} \Psi_{i}\right](A)=\cup_{i}\left[\Psi_{i}(A)\right]$ and $\left[\cap_{i} \Psi_{i}\right](A)=$ $\cap_{i}\left[\Psi_{i}(A)\right], A \in \mathcal{S}$. Then we have:

Proposition I [I]: a) $\mathcal{K}\left(\cap_{i \in I} \Psi_{i}\right)=\cap_{i \in I} \mathcal{K}\left(\Psi_{i}\right)$. b)

\footnotetext{
${ }^{4}$ The notation $\mathcal{K}(A \rightarrow \Psi(A))$ denotes $\mathcal{K}(\Psi)$.
} 
$\left.\mathfrak{K}\left(\bigcup_{i \in I} \Psi_{i}\right)=\bigcup_{i \in I} \mathfrak{K}\left(\Psi_{i}\right) . c\right) \Psi_{1}(A) \subseteq \Psi_{2}(A) \forall A \Rightarrow$ $\mathfrak{K}\left(\Psi_{1}\right) \subseteq \mathcal{K}\left(\Psi_{2}\right)$.

An SP system $\Psi$ is called increasing iff $A \subseteq B \Rightarrow \Psi(A)$ $\subseteq \Psi(B) \forall A, B$. Let $\Psi$ be a TI increasing SP system with domain $S$. If $\mathcal{S}^{d} \stackrel{\text { def }}{=}\left\{A^{c}: A \in \mathcal{S}\right\}$, the dual SP system of $\Psi$ is defined by $\Psi^{d}(A) \stackrel{\text { def }}{=}\left[\Psi\left(A^{c}\right)\right]^{c}, A \in \mathcal{S}^{d}$. Obviously, $\Psi^{d}$ is TI and increasing iff $\Psi$ is TI and increasing, respectively. The kernel of any TI increasing SP system has the following intriguing property:

Theorem 2 (Matheron [1] $)^{5}$ : Let $\Psi: \mathcal{S} \rightarrow \mathcal{P}(\boldsymbol{E})$ be a TI increasing SP system, where $S \subseteq \mathcal{P}(\boldsymbol{E})$ is closed under translation. Then, $\forall X \in \mathcal{S}$,

$$
\Psi(X)=\bigcup_{A \in \mathscr{K}(\Psi)} X \ominus A=\bigcap_{B \in \mathscr{K}^{(}\left(\Psi^{\mathrm{d} j}\right)} X \oplus \check{B} .
$$

\section{B. FP Systems}

We extended the kernel representation to FP systems as follows. Let the FP system $\psi$ be defined on a class $\mathcal{F}$ of extended real-valued input functions. Let $\Gamma$ be the corresponding umbra-processing system of $\psi$, i.e., $\Gamma[U(f)]$ $=U[\psi(f)], f \in \mathcal{F}$. Thus $\Gamma$ is defined on the (closed under translation) class $\mathcal{U}$ of the umbrae of all $f \in \mathcal{F}$. For each $f \in \mathcal{F}$, a translation of $U(f)$ by $z=(y, c) \in \boldsymbol{D} \times \boldsymbol{V}$ corresponds to shifting both the argument of $f$ by $y$ as well as the amplitude of $f$ by $c$; we call this the (vector) translation of $f(x), x \in D$, by $(y, c)$. Since $u$ is closed under set translation, $\mathcal{F}$ must be closed under function translation too. We say that

$$
\begin{gathered}
\psi \text { is TI } \Leftrightarrow \psi[f(x-y)+c]=[\psi(f)](x-y)+c \\
\forall f \in \mathcal{F}, \forall(y, c) \in \boldsymbol{D} \times \boldsymbol{V} .
\end{gathered}
$$

Clearly, $\psi$ is TI on $\mathcal{F}$ iff $\Gamma$ is TI on $\mathcal{U}$; then, the SP system $\Gamma$ has a kernel $\mathcal{K}(\Gamma)$. For each $f \in \mathcal{F}, U(f) \in \mathcal{K}(\Gamma)$ iff $(\overrightarrow{0}, 0) \in \Gamma[U(f)]=U[\psi(f)] \Leftrightarrow[\psi(f)](\overrightarrow{0}) \geq 0$. Thus, we define the kernel of the TI FP system $\psi$ as the following collection of input functions:

$$
\mathscr{K}(\psi) \stackrel{\text { def }}{=}\{f \in \mathcal{F}:[\psi(f)](\overrightarrow{0}) \geq 0\} .
$$

Obviously, there is a one-to-one correspondence between $\mathfrak{K}(\psi)$ and $\mathscr{K}(\Gamma)$, because for each $f \in \mathcal{K}(\psi)$ there is a unique umbra $U(f) \in \mathcal{K}(\Gamma)$ and vice versa. The kernel of FP systems has the following property (proven in the Appendix), which has no SP counterpart.

Proposition 2: If $\mathfrak{K}$ is the kernel of a TI FP system $\psi: \mathcal{F} \rightarrow \operatorname{ERV}(D)$ then,

$$
\begin{gathered}
\text { if } \boldsymbol{V}=\boldsymbol{R}, \quad f(x) \in \mathcal{K} \Leftrightarrow[f(x)+p] \in \mathcal{K} \forall p>0, \\
p \in \boldsymbol{R}, \\
\text { if } \boldsymbol{V}=\boldsymbol{Z}, \quad f(x) \in \mathcal{K} \Rightarrow[f(x)+p] \in \mathfrak{K} \forall p>0, \\
p \in \boldsymbol{Z} .
\end{gathered}
$$

From $\mathfrak{K}(\psi)$ we can uniquely reconstruct $\psi$, as the next result (proven in the Appendix) shows.

\footnotetext{
${ }^{5}$ Matheron's statement of Theorem 2 is less general because $S$ and $S^{d}$ are restricted to be equal, i.e., $\delta=\mathcal{S}^{d}=\boldsymbol{P}(\boldsymbol{E})$. However, this restriction is unnecessary as shown in $[9$, p. 121].
}

Proposition 3: Let $\mathcal{F} \subseteq$ ERV $(\boldsymbol{D})$ be closed under translation. Let $\mathcal{K}$ be any subclass of $\mathcal{F}$ satisfying (27). Then the system $\psi$ defined by

$$
[\psi(f)](x)=\sup \{a \in V: \underbrace{f(y+x)-a}_{\text {function of } y} \in \mathcal{K}\},
$$

is $\mathrm{TI}$, and its kernel is equal to $\mathscr{K}$.

Thus, there exists a one-to-one mapping from the space of all subclasses (the kernels) of $\mathfrak{F}$ that satisfy (27) onto the space of all TI systems defined on $\mathfrak{F}$.

Examples: Consider the FP erosion $\psi(f)=f \ominus g, f$ $\in \mathcal{F}$, by a given $g$. From (17), $f \ominus g(\overrightarrow{0})=$ $\inf _{y \in \operatorname{Spr}(g)}\{f(y)-g(y)\} \geq 0 \Leftrightarrow f(y) \geq g(y) \forall y$; hence, the kernel of this erosion-by-g FP system is

$$
\varepsilon(g)=\{f \in \mathcal{F}: f \geq g\} .
$$

Likewise, consider the FP dilation $\psi(f)=f \oplus g$. From (26) and (16), its kernel is

$$
\mathfrak{D}(g)=\left\{f \in \mathscr{F}: \sup _{y \in \operatorname{Spt}(f) \cap \operatorname{Spt}(\check{g})}[f(y)+g(-y)] \geq 0\right\} .
$$

The kernel of an FSP system $\phi$ on $\mathfrak{F}$ commuting with thresholding can be derived from the kernel of its SP counterpart $\Phi$. That is, from (26), (1), (21), and (22),

$$
\mathcal{K}(\phi)=\left\{f \in \mathcal{F}: T_{0}(f) \in \mathfrak{K}(\Phi)\right\} .
$$

For example, (31), (23), and (24) imply that the kernels of the FSP erosion and dilation by a set $B$ are

$$
\begin{aligned}
& \mathcal{K}(f \mapsto f \ominus B)=\left\{f \in \mathcal{F}: B \subseteq T_{0}(f)\right\}, \\
& \mathcal{K}(f \mapsto f \oplus B)=\left\{f \in \mathcal{F}: \breve{B} \cap T_{0}(f) \neq \varnothing\right\} .
\end{aligned}
$$

Assume henceforth that all TI FP systems $\psi$ of interest are nondegenerate: i.e., $\psi(f \equiv-\infty) \equiv-\infty, \psi(f \equiv$ $\infty) \equiv \infty$ (where $f \equiv c$ means that $f(x)=c \forall x$ ), and $\mathcal{K}(\psi)$ contains more elements than the single function $f$ $\equiv \infty$.

If $\left\{\psi_{i}: i \in I\right\}$ is an indexed family of TI FP systems, let us define their supremum and infimum superposition as the systems $\left[\vee_{i} \psi_{i}\right](f)=\vee_{i}\left[\psi_{i}(f)\right]$ and $\left[\wedge_{i} \psi_{i}\right](f)=$ $\wedge_{i}\left[\psi_{i}(f)\right], f \in \mathcal{F}$, respectively. Both superpositions correspond to parallel interconnection of systems. The kernel representation preserves the above types of superposition as well as ordering of systems, as stated below (see Appendix for proof).

Proposition 4: a) $\mathfrak{K}\left(\wedge_{i \in I} \psi_{i}\right)=\cap_{i \in I} \mathcal{K}\left(\psi_{i}\right)$. b) $\mathcal{K}(\psi)$ $=\cup_{i \in I} \mathcal{K}\left(\psi_{i}\right) \Rightarrow \psi=\vee_{i \in I} \psi_{i}$. If $I$ is finite, then $\left.\mathcal{K}\left(\vee_{i \in I} \psi_{i}\right)=\cup_{i \in I} \mathcal{K}\left(\psi_{i}\right) . c\right) \psi_{1}(f) \leq \psi_{2}(f) \forall f \in \mathcal{F} \Leftrightarrow$ $\mathscr{K}\left(\psi_{1}\right) \subseteq \mathscr{K}\left(\psi_{2}\right)$.

An FP system $\psi$ is called increasing iff $f \leq g \Rightarrow \psi(f)$ $\leq \psi(g) \forall f, g$. Thus, increasing systems are order-preserving. The increasing condition is of fundamental importance in image processing if we want the image operations to preserve order (contrast), or, equivalently, to be compatible with the spatial ordering of the opaque objects 
from which the image signals emanated. Moreover, increasing systems are consistent with the high-level vision models of gestalt psychology; in this field there is a strong belief that the perceptual processes underlying the visual interpretation of a scene are actually increasing systems. That is, as stated by Köhler [39], "Experienced order in space is always structurally identical with a functional order in the distribution of underlying brain processes." This is the principle of psychophysical isomorphism as it applies in the case of spatial order. For instance, let $f$ and $g$ be two image signals emanating from two opaque objects that partly cover each other. Then, as argued in [7], we perceive the signal $f \vee g$ through a high-level vision system $\psi$ and, because of the principle of psychophysical isomorphism, the transform, $\psi(f \vee g)$, of $f \vee g$ must dominate the max-superposition of the individual transforms; i.e., $\psi(f \vee g) \geq \psi(f) \vee \psi(g)$. This latter condition is equivalent to $\psi$ being increasing.

We extended Theorem 2 to FP systems as follows. Let $\psi$ be a TI FP system with domain $\mathcal{F}$. We define its dual (with respect to negation) FP system by $\psi^{d}(f) \stackrel{\text { def }}{=}$ $-\psi(-f), f \in \mathcal{F}^{d}$, where $(-f)(x)=-f(x)$ and $\mathcal{F}^{d} \stackrel{\text { def }}{=}$ $\{f:-f \in \mathcal{F}\} . \mathcal{F}^{d}$ is closed under translation iff $\mathfrak{F}$ is closed under translation. $\psi^{d}$ is TI and increasing iff $\psi$ is TI and increasing. The next result (proven in the Appendix) is an FP counterpart of Theorem 2.

Theorem 3: a) FP Systems: Let $\psi: \mathfrak{F} \rightarrow \operatorname{ERV}(\boldsymbol{D})$ be a TI increasing FP system, where $\mathfrak{F} \subseteq$ ERV $(\boldsymbol{D})$ is closed under translation. Then $\psi$ can be represented exactly as the pointwise supremum of erosions by all its kernel functions, and also as the pointwise infimum of dilations by all the reflected kernel functions of its dual system $\psi^{d}$; i.e., $\forall f \in \mathfrak{F}$,

$$
\psi(f)=\bigvee_{g \in \mathscr{K}(\psi)} f \ominus g=\bigwedge_{h \in \tilde{K}\left(\psi^{d}\right)} f \oplus \check{h} .
$$

b) FSP Systems: Let $\phi: \mathcal{F} \rightarrow \operatorname{ERV}(D)$, with $\mathcal{F}$ as in a), be a TI FSP system that commutes with thresholding, and $\Phi$ is its respective SP system. Then $\phi$ can be represented exactly as the pointwise supremum of erosions by all the kernel sets of $\Phi$, and also as the pointwise infimum of dilations by all the reflected kernel sets of the dual SP system $\Phi^{d}$; i.e., $\forall f \in \mathcal{F}$,

$$
\phi(f)=\bigvee_{A \in K(\Phi)} f \ominus A=\bigwedge_{B \in K_{(\Phi)}\left(\Phi^{d}\right)} f \oplus \breve{B} .
$$

\section{Basis Representations}

Theorems 2 and 3 may be theoretically interesting but they have no direct practical importance, because they require an infinite number of erosions to implement an increasing TI system, since the kerncl of such a system has an infinite number of elements [9]. Therefore, we were led to introduce the concept of the basis of such systems, which we defined as the collection of minimal kernel elements. If the basis is nonempty, then we may be able to exactly represent a system as a minimal (maybe finite) union of erosions using just the basis elements.
The kernel $\mathcal{K}(\Psi)$ of an SP system $\Psi$ defined on $S$ together with the ordering relation of set inclusion is a partially ordered set (poset). ${ }^{6}$ A kernel set-element is minimimal in $(\mathcal{K}(\Psi), \subseteq)$ iff it is not preceded (with respect to $\subseteq$ ) by any other kernel set. If $\Psi$ is also increasing, and $M \in \mathcal{K}(\Psi)$, then $\mathcal{E}(M)=\{A: A \supseteq M\} \subseteq \mathcal{K}(\Psi)$. In addition, $X \ominus A \subseteq X \ominus M$ for any set $X$ and $A \supseteq M$. Thus, in representing $\Psi$ as a union of erosions, the erosion by $M$ contains the erosions by any other kernel set in $\mathcal{E}(M)$, and, hence, it is the only one needed. We define the basis of any TI SP system $\Psi$ as the collection of its minimal kernel sets, denoted as

$$
\begin{array}{r}
B(\Psi) \stackrel{\text { def }}{=}\{M \in \mathcal{K}(\Psi):[A \in \mathcal{K}(\Psi) \text { and } A \subseteq M] \\
\Rightarrow A=M\} .
\end{array}
$$

The above discussion also applies to any FP increasing TI system $\psi$. Clearly, the pair $(\mathcal{K}(\psi), \leq)$ is a poset, where $\leq$ is the function ordering in (11). A kernel function-element is minimal in $(\mathcal{K}(\psi), \leq)$ iff it is not preceded (with respect to $\leq$ ) by any other kemel function. The basis of $\psi$ is the collection of its minimal kernel functions

$$
\begin{array}{r}
B(\psi) \stackrel{\text { def }}{=}\{g \in \mathscr{K}(\psi):[f \in \mathcal{K}(\psi) \\
\text { and } f \leq g] \Rightarrow f=g\} .
\end{array}
$$

In both cases the basis is a subcollection of the kerncl with infinitely fewer elements. As shown later, there are cases where the basis contains only a finite number of kernel elements.

At this point, two fundamental questions naturally arise: Does the basis exist? Can we represent the system only by its basis? In what follows we will answer both of these questions affirmatively. Toward this goal we had to restrict the general space of signals. Thus, instead of the most general set class $\mathcal{P}(\boldsymbol{E})$, now we select for signal representation the class of all closed subsets of $\boldsymbol{E}$, denoted by $\mathcal{C}(\boldsymbol{E})$. This is a natural compromise if we assume that each image object contains its boundary [1]. Therefore, all the threshold sets and the umbra of a function must be closed sets. The equivalent class of functions is the class of upper semicontinuous ${ }^{7}$ functions on $D$, denoted as USC $(\boldsymbol{D})$. That is, $f$ on $\boldsymbol{R}^{m}$ is upper semicontinuous iff $T_{a}(f)$ is a closed set in $\boldsymbol{R}^{m} \forall a \in \boldsymbol{R}$, or, equivalently, iff its umbra is closed set in $\boldsymbol{R}^{m+1}$. Discrete-domain signals are trivially upper semicontinuous because all their threshold sets are subsets of $\boldsymbol{Z}^{m}$ and hence closed.

\footnotetext{
${ }^{6}$ A relation " $\leq$ " on a set $S$ is called a partial ordering, and the pair ( $S$, $\leq$ ) is called a poset iff $\forall a, b, c \in S: 1$ ) $a \leq a, 2) a \leq b$ and $b \leq a$ imply $a=b$, and 3) $a \leq b$ and $b \leq c$ imply $a \leq c$. If ( $L, \leq$ ) is a poset and for all $a, b$ in $L$ we have $a \leq b$ or $b \leq a$, then ( $L, \leq$ ) is called a linearly ordered set.

${ }^{7} \mathrm{~A}$ function $f$ is upper semicontinuous at a point $x$ iff $f(x)=$ $\inf _{r>0}[\sup \{f(y): 0 \leq\|x-y\|<r\}]$ [40]. For example, if $c(x)$ is a continuous function, the function $g$ defined by $g(x)=c(x)$ for $x \neq x_{0}$ and $g\left(x_{0}\right)=c\left(x_{0}\right)+p$, is upper semicontinuous at $x_{0}$ if $p \geq 0$.
} 


\section{A. Existence of Minimal Elements}

Let $\left\{X_{n}\right\}_{n \geq 1}$ be any decreasing sequence of sets that converges monotonically to a limit set $X$, i.e., $X_{n+1} \subseteq X_{n}$ $\forall n$ and $X=\cap_{n} X_{n}$; we denote this by $X_{n} \downarrow X$. An increasing SP system $\Psi: \mathcal{C}(\boldsymbol{E}) \rightarrow \mathcal{C}(\boldsymbol{E})$ is called upper semicontinuous [1] iff $X_{n} \downarrow X$ implies that $\Psi\left(X_{n}\right) \downarrow \Psi(X)$. By using umbrae in the place of the sets $X_{n}$ we can extend these definitions to FP systems. That is, let $f_{n} \downarrow f$ denote a function convergence where $\left\{f_{n}\right\}_{n \geq 1}$ is a decreasing sequence (i.e., $f_{n+1} \leq f_{n} \forall n$ ) of functions that converges monotonically to a limit function $f=\wedge_{n} f_{n}$. Then, an increasing FP system $\psi$ is called upper semicontinuous iff $f_{n} \downarrow f$ implies that $\psi\left(f_{n}\right) \downarrow \psi(f)$ in USC $(\boldsymbol{D})$.

Upper semicontinuity amounts to requiring systems to he insensitive to fine details in the signal, since a physical resolution limit cannot be avoided. This, together with the already discussed importance of increasingness and translation-invariance, makes TI increasing upper semicontinuous systems an interesting class of signal operations. Such systems are "digitalizable," meaning that the transition between transforming continuous and discrete image objects satisfies a continuity condition [2]. Further, they play a central role in our work since the basis of a TI increasing SP or FP system exists (i.e., is nonempty) if the system is upper semicontinuous, as explained by the next theorem proven in the Appendix.

Theorem 4: Existence.

a) SP Systems: Let $\Psi: S \rightarrow \mathfrak{C}(E)$ be a TI increasing and upper semicontinuous SP system, where $\delta \subseteq \mathcal{C}(\boldsymbol{E})$ is closed under translation and any (possibly infinite) intersection. Then, the kernel of $\Psi$ has a minimal element.

b) FP Systems: Let $\psi: \mathcal{F} \rightarrow \operatorname{USC}(\boldsymbol{D})$ be a TI increasing and upper semicontinuous FP system, where $\mathcal{F} \subseteq$ USC $(D)$ is closed under translation and pointwise infimum. Then, the kernel of $\psi$ has a minimal element.

Upper semicontinuity and closedness of the systems' domains under $\cap$ or $\wedge$ are sufficient conditions for the existence of the basis. It remains to be found whether they are also necessary, or whether one can find other sufficient conditions for the existence of minimal elements.

\section{B. Representation by Minimal Elements}

We will show that any TI increasing and upper semicontinuous SP system $\Psi$ defined on $S \subseteq \mathcal{C}(\boldsymbol{E})$ is a union of erosions by its basis sets. To find a dual representation involving dilations, we should further restrict $S$ to discrete-domain signals. That is, both $\delta$ and $\mathcal{S}^{d}=\left\{A: A^{c} \in\right.$ $\mathcal{S}$ \} must be subsets of $\mathcal{C}(\boldsymbol{E})$. This is possible only if $\boldsymbol{E}$ $=\boldsymbol{Z}^{m}$. Further, since both $S$ and $\mathcal{S}^{d}$ must be closed under any intersection, they must also be closed under any union; they are also closed under translation. Therefore, in the case of dual representations we set $\delta=\delta^{d}=$ $\boldsymbol{P}\left(\boldsymbol{Z}^{m}\right)$. Next is our first theorem (proven in the Appendix) for representation by minimal elements.

Theorem 5 (Representation for SP systems):

a) All SP Systems: Let $\Psi: \mathcal{S} \rightarrow \mathcal{C}(E)$ be a TI increasing and upper semicontinuous SP system, where $\delta \subseteq$
$\mathcal{C}(\boldsymbol{E})$ is closed under translation and any intersection. Then $\Psi$ can be represented exactly as the union of erosions by all its basis sets; i.e., $\forall X \in \mathcal{S}$,

$$
\Psi(X)=\bigcup_{M \in \mathbb{B}(\Psi)} X \ominus M .
$$

b) Discrete SP Systems: Let $\Psi: \mathcal{P}\left(\boldsymbol{Z}^{m}\right) \rightarrow \boldsymbol{P}\left(\boldsymbol{Z}^{m}\right)$ be a discrete TI increasing and upper semicontinuous SP system. Then, if the dual system $\Psi^{d}$ is upper semicontinuous, $\Psi$ can be represented as the union of erosions by all its basis sets, and also as the intersection of dilations by all the reflected basis sets of $\Psi^{d}$; i.e., $\forall X \subseteq Z^{m}$,

$$
\Psi(X)=\bigcup_{M \in \mathbb{B}(\Psi)} X \ominus M=\bigcap_{N \in \mathbb{B}\left(\Psi^{d}\right)} X \oplus \check{N} .
$$

Since we consider only nondegenerate systems, the basis is a proper subset of the kernel. Hence, there is a proper subset $M$ of $\boldsymbol{E}$ belonging to the basis. Then, all the (infinite in number) sets $X$ in $S$ such that $M \subseteq X \subseteq E$ belong to the kernel but not to the basis. Thus, Theorem 5, compared with Matheron's Theorem 2, realizes the system by infinitely reducing the number of required erosions (or dilations). ${ }^{8}$ Examples are given in Section V.

Next we show that any TI increasing and upper semicontinuous FP system $\psi$, defined on $\mathfrak{F} \subseteq \operatorname{USC}(D)$, is a supremum of erosions by its basis functions. To find a dual representation for $\psi$, the domain $\mathfrak{F}^{d}=\{-f: f \in \mathfrak{F}\}$ of the dual FP system $\psi^{d}$ must be a subclass of USC $(D)$. But then both $f$ and $-f$ must be upper semicontinuous for all $f$ in $\mathfrak{F}$ and $\mathcal{F}^{d}$. Further, since both $\mathfrak{F}$ and $\mathcal{F}^{d}$ must be closed under pointwise infimum, they must also be closed under pointwise supremum. Hence, in the case of dual representations we select $\mathcal{F}=\mathcal{F}^{d}$ to be the class ERV $\left(\boldsymbol{Z}^{m}\right)$ of all extended real-valued functions defined on $\boldsymbol{Z}^{n}$. Next we present (see Appendix for proof) an FP counterpart of Theorem 5 .

Theorem 6 (Representation for FP systems):

a) All FP Systems: Let $\psi: \mathfrak{F} \rightarrow \operatorname{USC}(D)$ be a TI increasing and upper semicontinuous FP system, where $\mathcal{F}$ $\subseteq \mathrm{USC}(D)$ is closed under translation and pointwise infimum. Then $\psi$ can be represented exactly as the supremum of erosions by all its basis functions; i.e., $\forall f \in \mathfrak{F}$,

$$
\psi(f)=\underset{g \in \mathbb{B}(\psi)}{\vee} f \ominus g .
$$

b) Discrete FP Systems: Let $\psi: \operatorname{ERV}\left(\boldsymbol{Z}^{m}\right) \rightarrow \mathrm{ERV}$ $\left(\boldsymbol{Z}^{m}\right)$ be a discrete TI increasing and upper semicontinuous FP system. If the dual FP system $\psi^{d}$ is upper semicontinuous, then $\psi$ can be represented as the pointwise supremum of erosions by its basis functions, and also as the pointwisc infimum of dilations by the reflected basis functions of $\psi^{d}$; i.e., $\forall f$,

$$
\psi(f)={\underset{g \in B(\psi)}{\vee}}_{f} \theta g=\bigwedge_{h \in B(\psi d)} f \oplus \bar{h}
$$

\footnotetext{
${ }^{8}$ After we had presented and published Theorem 5 in [11] and [9], a theorem similar to Theorem 5a appeared subsequently in [35] only for discrete SP systems.
} 
c) All FSP systems: Let the FSP system $\phi: \mathcal{F} \rightarrow$ USC $(\boldsymbol{D})$, with $\mathcal{F}$ as in a), be TI and commute with thresholding. Let $\Phi$ be its respective SP system. Then $\phi$ can be represented exactly as the pointwise supremum of erosions by all the basis sets of $\Phi$; i.e., $\forall f \in \mathscr{F}$,

$$
\phi(f)=\underset{M \in(B(\Phi)}{\vee} f \ominus M .
$$

d) Discrete FSP Systems: Let the discrete FSP systcm $\phi: \operatorname{ERV}\left(\boldsymbol{Z}^{m}\right) \rightarrow \operatorname{ERV}\left(\boldsymbol{Z}^{m}\right)$ be TI and commute with thresholding. Let $\Phi$ be its respective SP system, and let its dual SP system $\Phi^{d}$ be upper semicontinuous. Then $\phi$ can be represented exactly as the pointwise supremum of erosions by the basis sets of $\Phi$, and also as the pointwise infimum of dilations by the reflected basis sets of $\Phi^{d}$; i.e., $\forall f$,

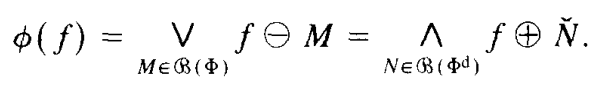

Observe that in Theorems $6 \mathrm{c}$ and $6 \mathrm{~d}$ the assumptions for an increasing and upper semicontinuous $\phi$ and for a TI increasing upper semicontinuous $\Phi$ are not needed, because they result from the commuting with thresholding. (See Lemma A1 in the Appendix.) For the same reasons as for SP systems, Theorem 6 realizes the FP system with infinitely fewer kernel functions than Theorem 3 . Further, for both SP and FP systems, if the basis is finite, then the systems can be realized by using only a finite number of erosions or dilations. Examples are given in Section V.

Concerning our general assumptions for the systems to be TI, increasing and upper semicontinuous, the next result $[9$, p. 139] provides some sufficient conditions:

Proposition 5: a) Any finite union or any intersection of TI increasing upper semicontinuous SP systems is a TI incrcasing upper semicontinuous system. The same is true for FP systems if we replace the finite union with maximum and the intersection with infimum. b) Any finite cascade of TI increasing upper semicontinuous systems is a TI increasing upper semicontinuous system.

It is known [1], [2] that erosion, dilation, opening, and closing by signals with compact support are TI increasing and upper semicontinuous systems. Hence, Proposition 5 implies that any finite maximum/minimum (parallel interconnection), or cascade (series interconnection) of such basic morphological filters is a TI increasing upper semicontinuous system; hence, the theory of minimal elements applies.

\section{EXAMPLES}

\section{A. Morphological Filters}

Unfortunately, our proof for the existence of minimal elements is not constructive; hence, there is not yet a systematic algorithm to find them. One technique that we employed for an SP system $\Psi$ is based on the fact that its minimal kernel elements are the smallest (with respect to $\subseteq$ ) solutions $X$ of the set equation $\overrightarrow{0} \in \Psi(X)$. However, finding these solutions is very system-dependent. For ex- ample, consider the SP erosion system $\Psi(X)=X \ominus A$ by a fixed set $A$. Then $\overrightarrow{0} \in X \ominus A \Leftrightarrow A \subseteq X$; the smallest $X \supseteq A$ is $A$. Hence, the erosion basis is

$$
B(X \mapsto X \ominus A)=\{A\} .
$$

Consider the SP dilation $\Phi(X)=X \oplus A$. Clearly, $\overrightarrow{0} \in$ $\Phi(X) \Leftrightarrow X \cap \breve{A} \neq \varnothing \Leftrightarrow-a \in X$ for some $a \in A$; the smallest such $X$ are the sets $\{-a\}, a \in A$. Hence, the dilation basis is

$$
B(X \mapsto X \oplus A)=\{\{-a\}: a \in A\} .
$$

Opening: Let $\Gamma(X)=X \bigcirc A=(X \ominus A) \oplus A$. Since $X \bigcirc A=\cup_{a \in A} X \ominus(A-a)=\cup_{a \in A}\{z:(A-a+z)$ $\subseteq X\}, \overrightarrow{0} \in \Gamma(X) \Leftrightarrow A-a \subseteq X$ for some $a \in A$; the smallest such $X$ are the sets $A-a, a \in A$. Thus the basis of the SP opening is

$$
\beta(X \mapsto X \bigcirc A)=\{A-a: a \in A\} .
$$

Closing: Let $\Delta(X)=X \ominus A=(X \oplus A) \ominus A$. Since $X \odot A=\bigcap_{a \in A} X \oplus(A-a)$, the kernel is $\mathcal{K}(\Delta)=$ $\{X: X \cap(\check{A}+a) \neq \varnothing \forall a \in A\}$. In this case we cannot find explicitly the basis elements, but we can find a fixed upper bound. That is, let $G \in \mathcal{K}(\Delta)$ and $F_{a}=G \cap(\check{A}$ $+a$ ). Obviously, $G \supseteq H=\cup_{a \in A} F_{a}$ and $H \in \mathcal{K}(\Delta)$. From Theorem Al in the Appendix, the kernel sets $G$ and $H$ contain a minimal element $M$. Then, $M \subseteq H \subseteq \cup_{a \in A}$ $\breve{A}+a=A \oplus \breve{A}$. Hence, the basis of the SP closing is

$$
\begin{aligned}
B(X \mapsto X \odot A)=\{M \subseteq A \oplus \check{A}: \overrightarrow{0} \in M \odot A \\
\text { and } M \text { is minimal }\} .
\end{aligned}
$$

Thus, the basis of discrete SP erosions, dilations, openings, and closings by a finite set $A$ with cardinality $|A|$ $=n \geq 1$ is finite. Specifically, the erosion has only one basis set, the dilation $n$, and the opening $n$ basis sets; the basis of the closing has cardinality smaller than $\mid P(A$ $\oplus \check{A}) \mid$. For analog morphological filters the formulas (44)-(47) are still valid. The only difference is that, since now $A \subseteq \boldsymbol{R}^{m}$, only the erosion will have a finite (one element) basis; the dilation, opening, and closing will have an infinite number of minimal elements.

Since $X \bigcirc A=\left(X^{r} \bigcirc \breve{A}\right)^{c}$, the dual SP system of the opening is the closing by $\breve{A}$. Then Theorem $5 b$ implies that

$$
X \bigcirc A=\bigcup_{a \in A} X \ominus(A-a)=\bigcap_{M \in \mathbb{B}(Y \mapsto Y \bullet \check{A})} X \oplus \breve{M} .
$$

When $A$ is 1-D, the basis of the closing by $A$ can be found more specifically; see Appendix for proof of the following.

Proposition 6: Consider any $n$-point convex 1-D set $A$ $=\{x \in Z: c \leq x \leq c+n-1\}, c \in Z, n \geq 1$. The basis of the discrete SP closing by $A$ consists exactly of the set $\{0\}$ and the $n(n-1) / 2$ sets $\{a, b\} \subseteq A \oplus \check{A}=\{y \in$ $Z:-n+1 \leq y \leq n-1\}$, where $a<0<b$ and $2 \leq$ $b-a \leq n$. 
If $A$ is a 1-D convex $n$-point set and $f$ is a sampled multilevel input signal, the obvious implementation of the FSP opening $f \bigcirc A$ as the cascade $(f \ominus A) \oplus A$ requires $2(n-1) \max / \min$ comparisons per output sample. From Theorem 6d, implementation of $f \bigcirc A$ (as max-of-minima) by the basis of the SP opening requires $\left(n^{2}-1\right)$ comparisons per output sample, whereas implementation of $f \bigcirc A$ (as min-of-maxima) by the basis of the SP closing requires $\left(n^{2}-n\right)$ such comparisons. In practice the window size $n$ is small; for such cases there may be applications (e.g., in VLSI hardware) where the basis implementation yielding a single local operation may be more favorable than the direct cascade that introduces delays and requires additional storage. Note also that the basis of the closing offers us a $[100 /(n+1)]$ percent reduction in the computations involved to find the opening. These savings are trivial for large $n$ but considerable for small $n$; e.g., if $n=3$, the savings are 20 percent and this 3-point opening provides signal smoothing comparable to a 5-point median filter, as cxplaincd in [13]. These 1-D openings/closings are not only useful for filtering 1-D signals, but 2-D signals as well; i.e., as investigated in [2], [41], performing openings or closing by four 1-D sets oriented at $0,45,90$, and 135 degrees and then taking the maximum of the four results provides 2-D smoothing without eliminating 1-D structures that reside in the 2-D image.

For 2-D structuring sets $A$ the basis of the opening is more advantageous than the closing; i.e., it is trivial to find the minimal elements of the opening using (46), whereas the minimal elements of the closing can still be found using (47) but the search procedure is usually more involved and their number grows fast with the size of $A$. For example, if $A=\{(0,0),(0,1),(1,0),(1,1)\}$, the basis of the opening by $A$ has only four 4-point sets, whereas the basis of the closing by $\boldsymbol{A}$ has eight sets: $\{0$, $0)\}$, two 2-point sets, four 3-point sets, and one 4-point set.

\section{B. Median and Rank Order Filters}

Median and rank order filters are nonlinear TI discrete filters that can suppress impulse (or speckle) noise from signals while preserving their edges; they have been used extensively for image and signal enhancement [42]-[45]. (See [42], [43] for reviews.) Let $f(x), x \in Z^{m}$, be an input sampled signal (function), and let $W \subseteq \boldsymbol{Z}^{m}$ be a finite window with $|W|=n$ points. For $r=1,2, \cdots, n$, the $r$ th rank order filter by $W$ is an FSP system $\phi$ with output $\phi(f)=\operatorname{RO}_{r}(f ; W)$, a function whose value at $x$ is the $r$ th largest number among the values $f(x+y), y \in W$. The corresponding SP system of $\phi$ is a rank order filter $\Phi(X)=\mathrm{RO}_{r}(X ; W)$ for discrete sets $X \subseteq Z^{m}$, where

$$
\mathrm{RO}_{r}(X ; W) \stackrel{\text { def }}{=}\left\{p \in Z^{m}:|X \cap W+p| \geq r\right\} .
$$

If $n$ is odd, for $r=(n+1) / 2$ we have the median filter of a function $f$ (or set), denoted as med $(f ; W)$.

Rank order filters $\phi$ commute with thresholding, as investigated in [6], [37], [2], [9], [13] and [42, ch. 5, 6].
Hence (see Lemma A1 in the Appendix), they are increasing and upper semicontinuous TI systems. The basis of $\Phi$ is [13]

$$
\beta\left(X \mapsto \operatorname{RO}_{r}(X: W)\right)=\{M \subseteq W:|M|=r\},
$$

and has $n ! / r !(n-r)$ ! elements. The dual SP system of $\Phi$ is the $(n-r+1)$ th rank order filter by $W$. Then Theorem $6 \mathrm{~d}$ yields:

$$
\begin{aligned}
& {\left[\operatorname{RO}_{r}(f ; W)\right](x)=} \max _{A \subseteq W}\left[\min _{y \in A}\{f(x+y)\}\right] \\
&|A|=r \\
&= \min _{B \subseteq W}\left[\max _{y \in B}\{f(x+y)\}\right] . \\
&|B|=n-r+1
\end{aligned}
$$

For cxample, lct $W=\{(0,0),(0,1),(1,0),(-1$, $0),(0,-1)\}$ be the 5 -point discrete disk of radius 1 . Then the basis of the 2-D SP median by $W$ has only ten elements $A_{k}, 1 \leq k \leq 10$ : all the 3-point subsets of $W$. Thus,

$$
\begin{gathered}
\operatorname{med}\left\{\begin{array}{l}
f(x-1, y), f(x, y), f(x+1, y) \\
f(x, y-1), f(x, y+1)
\end{array}\right\} \\
=\max _{k=1}^{10}\left[\min _{(i, j) \in A_{k}}\{f(x+i, y+j)\}\right]
\end{gathered}
$$

for any signal $f(x, y)$ on $Z^{2}$; we can also interchange min and max in (52).

The implications of (51) are profound because they enable us to express any median and rank order filter via a closed formula involving only max-min of prespecified sets of numbers without requiring any sorting. For large window sizes $|W|$ this max-min realization may have higher computational complexity than other fast sorting schemes because the number of minimal elements grows fast. However, for small windows $W$ implementation of rank order filters via its basis is quite attractive in practical applications. For example, at the Harvard Robotics Lab we are currently investigating this max-min implementation of median filtering by the 5-pixel window $W$ of (52), which allows us to perform 2-D median filtering in real-time on Max Video pipelined image processing architectures manufactured by Data Cube Inc. These architectures perform a local max-min (by any subwindow of a $3 \times 3$ neighborhood) of $512 \times 512$-pixel images in a $1 / 30$ th of a second, but they cannot do sorting. Thus, performing the median filtering by sending the image to the host computer for the necessary sorting would require at least a few minutes per image frame. However, the basis implementation of a median filter by the 5-pixel circular 2-D window requires a maximum of ten local minima, which can be done on the Data Cube and requires only half a second per image frame. For a median by a $3 \times 3$-pixel window, which has 126 minimal elements of 5 points each, the basis implementation would require ap- 
proximately 4 seconds to do the local minima and $4 \mathrm{sec}$ onds for the maximum.

Wendt et al. [38] defined stack filters as a generalization of rank order filters based on threshold decomposition and Boolean functions. In [13] it was shown that stack filters are all the finite max-min operations, and their basis representation is equivalent to representing their defining Boolean function via irreducible sum-of-products expressions.

Next we introduce a generalization of rank order filters in order to relate them to FP erosions and dilations that use a multilevel structuring function $g(x)$ with finite support $W=\operatorname{Spt}(g)$. For $1 \leq r \leq n=|W|$, we define the $r$ th generalized rank order filter by $g$ as the FP system $\psi$ whose output is

$$
\begin{aligned}
\psi(f) & =\left[\operatorname{RO}_{r}(f ; g)\right](x) \\
= & r \text { th largest of } f(x+y)+g(y), \quad y \in W .
\end{aligned}
$$

Note that the first ( $r=1)$ rank order filter by $g$ coincides with the discrete FP dilation by $\breve{g}$, whereas the last ( $r=$ $n$ ) rank order filter is the FP erosion by $-g$. If $g$ is binary, i.e., 0 on $W$, these filters reduce to the simple rank order filters of the previous discussion. Assume now that $g$ is not binary. These general rank order filters do not commute with thresholding. Their basis consists of $n ! / r !(n$ $-r)$ ! input functions $h$ such that $h(y)=-g(y)$ for $r$ points $y \in W$ and $\mid$ Spt $(h) \mid=r$. For example, consider the generalized median ( $n=3, r=2$ ) of 1-D signals $f(x)$ by the function $g(x)$ defined as $g(-1)=1, g(0)$ $=4, g(1)=-2$, and $g(x)=-\infty \forall x \notin W=\{-1,0$, $1\}$. Its basis has only 3 functions $h_{1}, h_{2}, h_{3}$ :

$$
\begin{aligned}
h_{1}(-1) & =-1, h_{1}(0)=-4, \operatorname{Spt}\left(h_{1}\right)=\{-1,0\} \\
h_{2}(-1) & =-1, h_{2}(1)=2, \operatorname{Spt}\left(h_{2}\right)=\{-1,1\} \\
h_{3}(0) & =-4, h_{3}(1)=2, \operatorname{Spt}\left(h_{3}\right)=\{0,1\} .
\end{aligned}
$$

Then med $(f ; g)=\vee_{i=1}^{3} f \ominus h_{i}$ due to Theorem 6a; i.e.,

$$
\begin{aligned}
\text { med } & \{f(x-1)+1, f(x)+4, f(x+1)-2\} \\
= & \max \left\{\begin{array}{l}
\min \{f(x-1)+1, f(x)+4\} \\
\min \{f(x-1)+1, f(x+1)-2\} \\
\min \{f(x)+4, f(x+1)-2\}
\end{array}\right\} .
\end{aligned}
$$

Simple medians by $W$ behave like FSP openings/closings [13]. Hence, the practical usefulness of the generalized medians by $g$ is similar to the FP openings/closings by $g$.

\section{Linear Shift-Invariant Systems}

Let $h(x), x \in \boldsymbol{D}$, be the impulse response of a classical linear shift-invariant system $\psi(f)=h * f, f \in \mathcal{F}$, where * denotes linear convolution (analog or discrete) and $F$ is a closed under translation class of functions with range $\boldsymbol{R}$. $\psi$ is translation-invariant in the sense we have defined, if it passes constant inputs unchanged, or equivalently if $h$ has area equal to one. Then the kernel of $\psi$ is $\mathfrak{K}(\psi)=$ $\{g \in \mathfrak{F}: h * g(\overrightarrow{0}) \geq 0\} . \psi$ is increasing iff $h$ is nonnegative everywhere [12]. Due to the linearity of $\psi$, the dual filter of $\psi$ is identical with $\psi$; i.e., $\psi^{d}(f)=-\psi(-f)$ $=\psi(f)$.

For example, if $h(x)=(1 / \sigma \sqrt{2 \pi}) e^{-\left(x^{2} / 2 \sigma^{2}\right)}, x \in \boldsymbol{R}$, is a Gaussian, then $h(x) \geq 0 \forall x$ and $\int_{R} h(x) d x=1$; hence Theorem 3 yields

$$
\begin{aligned}
& \frac{1}{\sigma \sqrt{2 \pi}} \int_{-\infty}^{+\infty} f(y) e^{-(x-y)^{2} / 2 \sigma^{2}} d y \\
& \quad=\sup _{g \in \mathcal{K}}\{f \ominus g(x)\}=\inf _{g \in \mathscr{K}}\{f \oplus \breve{g}(\mathrm{x})\},
\end{aligned}
$$

where $\mathcal{K}=\left\{g \in \mathcal{F}: \int_{-\infty}^{+\infty} g(y) e^{-y^{2} / 2 \sigma^{2}} d y \geq 0\right\}$. Note that the restricting conditions that the impulse response be everywhere nonnegative and its area be equal to one can be relaxed [12].

If $\psi$ is a discrete linear TI increasing system whose impulse response $h(n), n \in Z^{m}$, has a finite support $\{n: h(n)$ $\neq 0\}$ (this latter assumption makes $\psi$ upper semicontinuous), then $\psi$ has a basis given by [12]

$$
\begin{aligned}
\leftrightarrow(\psi)= & \{g \in \mathcal{F}: h * g(\overrightarrow{0})=0, \\
& \text { and } g(n)--\infty \Leftrightarrow h(-n)=0\} .
\end{aligned}
$$

For example, let $h(n)=a \delta(n)+(1-a) \delta(n-1)$, $n \in Z$, where $0<a<1$ and $\delta(n)$ is the discrete unit impulse. $\psi$ is a moving average filter; its basis functions are $g(n), n \in \boldsymbol{Z}$, where $g(0)=r \in \boldsymbol{R}, g(-1)=-a r /$ $(1-a)$, and $g(n)=-\infty$ for $n \neq 0,-1$. Then Theorem $6 \mathrm{~b}$ yields

$$
\begin{aligned}
a f(n) & +(1-a) f(n-1) \\
= & \sup _{r \in R}\left[\min \left\{f(n)-r, f(n-1)+\frac{a r}{1-a}\right\}\right] .
\end{aligned}
$$

Since a supremum of erosions involves only additions and max-min comparisons, if we can use only a finite number of the required erosions to realize a linear TI system (e.g., by quantizing and bounding the range of input signals) and quantify the approximation errors, these morphological representations may become useful in implementing linear systems without using multiplications. These issues are still under investigation.

\section{Window-Transforms for Shape Recognition}

Let $A$ be a fixed compact set and let $W$ be another compact set containing $A$ such that the set difference $W \backslash A$ serves as a local background (narrow ring) around $A$. Then the hit-or-miss transform [2] $(X \ominus A) \cap\left[X^{c} \ominus(W \backslash A)\right]$ provides the set of points $p$ at which $A$ "fits exactly" inside an input set $X$; i.e., $A+p \subseteq X$ and $(W \backslash A)+p \subseteq$ $X^{c}$. Crimmins and Brown [8] proved that this shape recognition transform is the prototype for a large class of binary or graytone image transforms, called window-tranforms ( $W$-transforms), defined below.

Theorem 7 [8]: Let $W \subseteq Z^{2}$. An SP system $\Psi$ is called a $W$-transform iff there exists $\mathcal{H} \subseteq \mathcal{P}(W)$ such that, $\forall X$ 


$$
\begin{aligned}
& \subseteq Z^{2}, \\
&
\end{aligned}(X)=\left\{p \in Z^{2}:(W \cap X-p) \in \mathcal{H}\right\} .
$$

If $W$ is finite, then, $\forall X \subseteq Z^{2}$,

$$
\Psi(X)=\bigcup_{A \in \mathscr{K}}(X \ominus A) \cap\left[X^{c} \ominus(W \backslash A)\right] .
$$

Thus $\Psi(X)$ in (59) is the set of points $p$ at which at least one of the patterns $A$ in $\mathcal{H}$, shifted at location $p$, fits exactly inside $X$. Next we analyze the kernel and basis of $W$-transforms.

Any $W$-transform $\Psi$ is uniquely defined by its class $\mathcal{H}$ via (58). Since $\Psi$ is obviously a TI system, it can also be defined by its kernel. Given a $W$-transform $\Psi$ defined by (58), it can be shown that

$$
\mathfrak{H}=\{A \subseteq W: \overrightarrow{0} \in \Psi(A)\} \subseteq \mathfrak{K}(\Psi) .
$$

If $W$ is infinite, i.e., $W=Z^{2}$, then $\mathfrak{H}=\mathscr{K}(\Psi)$; further, the definition of $\Psi$ through (58) becomes identical to the representation of $\Psi$ via its kernel. Therefore, the kernel representation of TI systems is more general than Theorem 7 because $W$-transforms are just a special class of TI systems.

Further, an increasing upper semicontinuous $W$-transform $\Psi$ can be represented more efficiently (computationally) as a union of erosions by its basis elements than with (59). That is, (59) represents $\Psi$ as a union of hit-or-miss transforms (more complex than erosions) by all the sets in $\mathfrak{H}$, which is larger and contains the basis of $\Psi$. To see this, let $F \subseteq G \subseteq W$ with $F \neq G$ and $0 \in \Psi(F) \subseteq$ $\Psi(G)$. Then both $F$ and $G$ of $W$ belong to $\mathfrak{K}$, but only $F$ may belong to $B(\Psi)$, because $G$ is not a minimal element of $\mathcal{K}(\Psi)$. For example, let $W$ be the 5-point 2-D window of (52) and consider the SP system $\Psi(X)=\operatorname{med}(X ; W)$, $X \subseteq Z^{2}$. Then $\Psi$ is a $W$-transform whose defining class is $\mathfrak{K}=\{A \subseteq W: 3 \leq|A| \leq 5\}$. Thus, $\mathfrak{H}$ consists of 16 subsets of $W$; these subsets are the patterns that the $W$-transform recognizes. The basis of $\Psi$ is $B(\Psi)=\{A$ $\subseteq W:|A|=3\}$. Thus $B(\Psi)$ contains only 10 sets, and Theorem 5 represents $\Psi$ as the union of 10 erosions by these basis sets. However, Theorem 7 represents $\Psi$ as the union of 16-hit-or-miss transforms by the elements of $\mathcal{H}$, and each hit-or-miss transform is the intersection of two erosions; hence, the latter representation is computationally less efficient because it requires a larger number of more complex morphological operations.

\section{Conclusions}

The major contributions of this paper are the development of: 1) a kernel for translation-invariant increasing systems processing multilevel signals and their representation as a sup-of-erosions by kernel functions; 2) a basis of the kernel, and representations of translation-invariant increasing and upper semicontinuous systems for multilevel (binary) signals as a supremum (union) of erosions by basis signals; 3 ) results (1) and (2) for systems processing signals with both discrete and continuous domain; 4) analysis of various nonlinear and linear systems by their kernel or basis.
For future work, more systematic general algorithms are needed for obtaining the basis. In addition, if some direct links or analytic criteria are found between desirable properties of systems and their basis, then the design of new useful systems can be approached through their minimal elements representation.

\section{Appendix}

\section{Proof of Proposition 2}

Let $f \in \mathfrak{K}$; then $[\psi(f)](\overrightarrow{0}) \geq 0$. For all (real or integer) $p>0,[\psi(f)](\overrightarrow{0})+p>0$ and hence $[f(x)+$ $p] \in \mathcal{K}$. Conversely (for the case $\boldsymbol{V}=\boldsymbol{R}$ ), let $[f(x)+$ $p] \in \mathfrak{K} \forall p>0$ and $g=\psi(f)$. Then $g(\overrightarrow{0}) \geq-p$, which implies $\overrightarrow{0} \in T_{a=-p}(g) \forall a<0$. From (3) we get $T_{0}(g)$ $=\left[\cap_{a<0} T_{a}(g)\right] \supseteq\{\overrightarrow{0}\}$. Hence $g(\overrightarrow{0}) \geq 0$, and thus $f$ $\in \mathscr{K}$.

Q.E.D.

\section{Proof of Proposition 3}

Let $f_{(z, c)}(x)$ denote the translate $f(x-z)+c$ of $f(x)$ by $(z, c)$. From (28), $\left[\psi\left(f_{(z, c)}\right)\right](x)=\sup$ $\left\{a: f_{(z-x . c-a)} \in \mathcal{K}\right\}=\sup \left\{t+c: f_{(z-x,-t)} \in \mathcal{K}\right\}$, with $t=a-c$. Thus, $\left[\psi\left(f_{(z, c)}\right)\right](x)=c+\sup$ $\left\{t: f_{(z-x,-t)} \in \mathcal{K}\right\}=c+[\psi(f)](x-z)$. Hence, $\psi$ is TI.

Let $\mathcal{K}(\psi)$ be the kernel of $\psi$. For each $f \in \mathcal{F}$, let $A=$ $\left\{a \in V: f_{(0,-a)} \in \mathcal{K}\right\}$. First, let $f=f_{(\overrightarrow{0}, 0)} \in \mathcal{K}$. Then $0 \in$ $A \Rightarrow[\psi(f)](\overrightarrow{0}) \geq 0 \Rightarrow f \in \mathcal{K}(\psi)$; hence $\mathcal{K} \subseteq \mathcal{K}(\psi)$. Conversely, let $f \in \mathfrak{K}(\psi)$, which implies $[\psi(f)](\overrightarrow{0})=$ $\sup (A) \geq 0$. Assume first $\boldsymbol{V}=\boldsymbol{R}$. If $\sup (A)>0$, there is $a>0$ such that $f_{(\overrightarrow{0},-a)} \in \mathcal{K}$; then (27) yields $\left[f_{(\overrightarrow{0},-a)}\right.$ $+a]=f \in \mathcal{K}$. If $\sup (A)=0, a \in A \forall a<0$; then $f_{\left({ }_{0}, p\right)} \in \mathcal{K} \forall p=-a>0$, which implies $f \in \mathfrak{K}$. Likewise, if $V=Z$, then sup $(A) \geq 0 \Rightarrow$ there exists $a \geq 0$ in $A$ $\Rightarrow f_{(0,-a)} \in \mathfrak{K} \Rightarrow f_{(0,-a)}+a=f \in \mathcal{K}$. Thus $\mathfrak{K}(\psi) \subseteq \mathfrak{K}$ $\subseteq \mathcal{K}(\psi) \Rightarrow \mathcal{K}=\mathfrak{K}(\psi)$.

Q.E.D.

\section{Proof of Proposition 4}

a) $f \in \mathscr{K}\left(\wedge_{i} \psi_{i}\right) \Leftrightarrow \inf _{i}\left\{\left[\psi_{i}(f)\right](\overrightarrow{0})\right\} \geq 0 \Leftrightarrow$ $\left.\left[\psi_{i}(f)\right](\overrightarrow{0})\right\} \geq 0 \forall i \Leftrightarrow f \in \mathfrak{K}\left(\psi_{i}\right) \forall i \Leftrightarrow f \in \cap_{i} \mathfrak{K}\left(\psi_{i}\right)$.

b) If $\mathcal{K}(\psi)-\cup_{i} \mathcal{K}\left(\psi_{i}\right)$, then, from $(28),[\psi(f)](x)$ $=\sup \left\{a:\left[\psi_{i}(f)\right](x) \geq a\right.$, some $\left.i\right\}=\sup _{i}\left\{\left[\psi_{i}(f)\right]\right.$ $(x)\}$. Thus, $\psi=\vee_{i} \psi_{i}$. If $I$ is finite, then $\mathfrak{K}\left(\vee_{i} \psi_{i}\right)=$ $\left\{f: \max _{i}\left\{\left[\psi_{i}(f)\right](\overrightarrow{0})\right\} \geq 0\right\}=\left\{f:\left[\psi_{i}(f)\right](\overrightarrow{0}) \geq\right.$ 0 , some $i\}=\left\{f: f \in \mathcal{K}\left(\psi_{i}\right)\right.$, some $\left.i\right\}=\bigcup_{i} \mathcal{K}\left(\psi_{i}\right)$.

c) Let $\Gamma_{1}, \Gamma_{2}$ be the respective umbra-processing systems of $\psi_{1}, \psi_{2}$. Then, $\mathcal{K}\left(\psi_{1}\right) \subseteq \mathcal{K}\left(\psi_{2}\right) \Leftrightarrow \mathcal{K}\left(\Gamma_{1}\right) \subseteq$ $\mathcal{K}\left(\Gamma_{2}\right) \Leftrightarrow \Gamma_{1}[U(f)] \subseteq \Gamma_{2}[U(f)] \Leftrightarrow U\left[\psi_{1}(f)\right] \subseteq$ $U\left[\psi_{2}(f)\right] \Leftrightarrow \psi_{1}(f) \leq \psi_{2}(f) \forall f$.

Q.E.D.

For Theorem $3 b$ we necd the following.

Lemma A1: Let $\phi: \mathfrak{F} \rightarrow \mathcal{G}$ be an FSP system with $\mathcal{F}$, $\mathcal{G} \subseteq E R V(D)$, and let $\Phi$ be its respective SP system. If $\phi$ commutes with thresholding, then:

a) Both $\phi$ and $\Phi$ are increasing [12] .

b) If $\mathcal{G}=U S C(D)$ and $\mathcal{F} \subseteq \mathcal{G}$ is closed under pointwise infimum, then both $\phi$ and $\Phi$ are upper semicontinuous [12].

c) If $\mathcal{F}$ is closed under translation, then $\phi$ is TI iff $\Phi$ is TI. 
Proof. c) Let $\phi$ be TI and let $A \subseteq D$. If $\chi_{A}(z)$ is the characteristic function of $A$, then $\phi\left[\chi_{A}(z-y)\right]=$ $\left[\phi\left(\chi_{A}\right)\right](z-y) \forall y \in D$. Thus $\Phi(A+y)=\Phi(A)+y$, which implies that $\Phi$ is TI. Conversely, let $\Phi$ be TI and let $g=\phi(f)$ for some $f(x) \in \mathscr{F}$. We use the fact that $T_{a}[f(x-y)+c]=\left[T_{a-c}(f)\right]+y, \forall(y, c) \in D \times$ $V$. Then, $\forall a, T_{a}[\phi(f(x-y)+c)]=\Phi\left(\left[T_{a-c}(f)\right]+\right.$ $y)=\Phi\left[T_{a-c}(f)\right]+y=\left[T_{a-c}(g)\right]+y=T_{a}[g(x-$ $y)+c]$. Thus, $\phi[f(x-y)+c]=g(x-y)+c$, and hence $\phi$ is TI.

Q.E.D.

\section{Proof of Theorem 3:}

a) Let $\mathfrak{K}=\mathfrak{K}(\psi)$. Since $\psi$ is increasing and TI, if $g$ $\in \mathfrak{K}, h \in \mathcal{F}$, and $h \geq g$, then $h \in \mathfrak{K}$, because [ $\psi(h)]$ $(\overrightarrow{0}) \geq[\psi(g)](\overrightarrow{0}) \geq 0$. Thus, see $(29), \varepsilon(g) \subseteq \mathcal{K}$ $\forall g \in \mathfrak{K}$; hence, $\cup_{g \in \mathcal{K}} \mathcal{E}(g) \subseteq \mathcal{K}$. Also, since $\{g\} \subseteq$ $\mathcal{E}(g), \mathcal{K}=\cup_{g \in \mathcal{K}}\{g\} \subseteq \cup_{g \in \mathcal{K}} \mathcal{E}(g)$. Thus $\mathcal{K}=$ $\cup_{g \in \mathcal{K}} \mathcal{E}(g)$. Proposition $4 \mathrm{~b}$ then yields

$$
\psi(f)=\bigvee_{g \in \mathcal{K}} f \ominus g, \quad \forall \int \in \mathcal{F} .
$$

Applying this result to the TI increasing system $\psi^{d}$, we have that

$$
\begin{aligned}
\psi(f) & =-\left[\psi^{d}(-f)\right]=-\bigvee_{h \in \mathcal{K}^{\left(\psi^{d}\right)}}(-f) \ominus h \\
& =\hat{h}_{\mathcal{K}\left(\psi^{d}\right)} f \oplus \breve{h},
\end{aligned}
$$

because $(-f) \ominus h=-(f \oplus \breve{h})$. Thus the proof of (34) is complete.

b) $\Phi$ is defined on the (closed under translation) class $\mathcal{S}$ of the threshold sets of all $f \in \mathscr{F}$. Because of Lemma $\mathrm{A} 1, \Phi$ is $\mathrm{TI}$ and increasing. From Theorem 2, $\forall a \in V$,

$$
\begin{aligned}
T_{a}[\phi(f)] & =\Phi\left[T_{a}(f)\right]=\bigcup_{A \in \mathscr{K}(\Phi)}\left[T_{a}(f)\right] \ominus A \\
& =\bigcap_{B \in \mathscr{K}\left(\Phi^{d}\right)}\left[T_{a}(f)\right] \oplus \check{B} .
\end{aligned}
$$

Since, $\left[T_{a}(f)\right] \ominus A=T_{a}(f \ominus A)$ and $\left[T_{a}(f)\right] \oplus \breve{B}=$ $T_{a}(f \oplus \breve{B}),(61)$ completes the proof of (35) because of $(8)$ and (9).

Q.E.D.

\section{Proof of Theorem 4}

a) Let $\mathcal{K}$ be the kernel of $\Psi$. Since $\Psi$ is nondegenerate, the poset $(\mathcal{K}, \subseteq)$ has an infinite number of elements $[9$, p. 130]. Let $\mathscr{L}=\left\{X_{i}: i \in I\right\}$ be any linearly ordered subset of $\mathcal{K}$, where $I$ is some index set. Then $X_{i} \subseteq X_{j}$ or $X_{i} \subseteq X_{i}$ for any $i, j \in I$. The set $X=\cap_{i \in I} X_{i}$ belongs to $\varsigma$ and is clearly a lower bound of $\mathcal{L}$. If $X=X_{i}$ for some $i$, then obviously $X \in \mathcal{K}$. Assume now that $X \neq X_{i} \forall i \in I$; this implies that $I$ is infinite and that $\mathscr{L}$ contains an infinite number of distinct elements. Then, with respect to the hitor-miss topology [1] of $\mathcal{C}(\boldsymbol{E})$, it can be shown that $X$ is a limit point of the infinite set $\mathcal{L}$. Hence, since $\mathcal{C}(\boldsymbol{E})$ is a compact, Hausdorff topological space with a countable base, there is an infinite sequence $\left\{X_{n}\right\}_{n \geq 1}$ of distinct $X_{n}$ $\in \mathscr{L}$ converging to $X$. Let $Y_{1}=X_{n}$ for some integer $n_{1}$. Assume that for $1,2, \cdots, k \geq 1$, increasing positive integers $n_{1}, n_{2}, \cdots, n_{k}$ have been chosen such that $Y_{j}=$ $X_{n}, 1 \leq j \leq k$, and $Y_{1} \supseteq Y_{2} \supseteq \cdots \supseteq Y_{k}$. Then there exists integer $n_{k+1}>n_{k}$ such that $Y_{k+1}=X_{n_{k+1}} \subseteq Y_{k}$, because otherwise $X_{n} \supseteq Y_{k} \forall n>n_{k}$ which implies that $\lim X_{n} \supseteq Y_{k}$ and hence $\lim X_{n} \neq X$. Thus, by induction on $k$, we can find a decreasing set sequence $\left\{Y_{k}=\right.$ $\left.X_{n k}\right\}_{k \geq 1}$ that is a subsequence of $\left\{X_{n}\right\}$. Hence, $Y_{k} \downarrow X=$ $\lim Y_{k}$. Since $\Psi$ is increasing and upper semicontinuous, $Y_{k} \downarrow X \Rightarrow \Psi\left(Y_{k}\right) \downarrow \Psi(X)=\bigcap_{k} \Psi\left(Y_{k}\right)$. Further, $Y_{k} \in \mathcal{K}$ $\forall k \Rightarrow \overrightarrow{0} \in \Psi\left(Y_{k}\right) \forall k \Rightarrow \overrightarrow{0} \in \Psi(X) \Rightarrow X \in \mathcal{K}$. Thus every linearly ordered subset of $\mathcal{K}$ has a lower bound in $\mathcal{K}$. Therefore, from Zorn's lemma [40] we infer that $\mathcal{K}$ has a minimal element.

b) Let $\Gamma$ be the equivalent umbra-processing system of $\psi$, defined on $\mathcal{U}=\{U(f): f \in \mathfrak{F}\}$. Then $\mathcal{U} \subseteq \mathcal{C}(\boldsymbol{E})$ is closed under translation and intersection, and $\Gamma$ is TI increasing and upper semicontinuous $[9$, p. 133]. By applying Theorem $4 \mathrm{a}$ to $\Gamma$ we infer that the poset $(\mathfrak{K}(\Gamma), \subseteq)$ has a minimal element, call it $V$; then there exists $g \in$ $\Re(\psi)$ such that $V=U(g)$. Due to (11) and the one-toone correspondence between $\mathscr{K}(\Gamma)$ and $\mathscr{K}(\psi)$, the poset $(\mathcal{K}(\psi), \leq)$ is isomorphic to $(\mathcal{K}(\Gamma), \subseteq)$. Hence, $g$ is a minimal element of $(\mathcal{K}(\psi), \leq)$.

Q.E.D.

For Theorems 5 and 6 we need to prove the following.

Theorem Al: Let $\Psi$ and $\psi$ be an SP and FP system satisfying the assumptions of Theorems $4 \mathrm{a}$ and $4 \mathrm{~b}$, respectively. Then, for any $A \in \mathscr{K}(\Psi)$, there exists a minimal kernel set $M \in B(\Psi)$ such that $A \supseteq M$. Likewise, for any $f \in \mathfrak{K}(\psi)$, there exists a minimal kernel function $g \in B(\psi)$ such that $f \geq g$.

Proof: SP systems: let $\mathcal{K}=\mathcal{K}(\Psi)$. If $A \in \mathcal{K}=$ $\cup_{B \in \mathcal{K}} \mathcal{E}(B)$, then $A \in \mathcal{E}(B)$, and hence $A \supseteq B$ for some $B \in \mathfrak{K}$. Likewise, $B \in \mathcal{E}(C) \Rightarrow B \supseteq C$ for some $C \in \mathfrak{K}$. Hence, for any $A \in \mathcal{K}$ we can find a decreasing sequence of kernel sets $A \supseteq B \supseteq C \supseteq \cdots$ and so on. Thus we can find a linearly ordered subset of $\mathcal{K}$, call it $\mathcal{L}$, that contains $A$. From Hausdorff's maximality principle [40], there is a maximal linearly ordered subset $\mathscr{K}$ of $\mathscr{K}$ containing $\mathfrak{L}$. Let $M=\cap \mathfrak{K}$ be the intersection of all sets of $\mathfrak{M}$. Then $A \supseteq \cap \mathscr{L} \supseteq \cap \mathfrak{K}=M$. In proving Theorem 4a we showed that $M$ is an element of $\mathcal{K}$. In addition, $M$ is a minimal element of $\mathscr{K}$, because otherwise there is a set $Y \neq M$ in $\mathscr{K}$ such that $Y \subseteq M$. But then $\mathfrak{K} \cup\{Y\}$ is a linearly ordered subset of $\mathfrak{K}$ which contains properly $\mathfrak{N}$ : contradiction, because $\mathfrak{N}$ is maximal. Hence, $M \in$ $B(\Psi)$ and $A \supseteq M$.

FP Systems: For any $f \in \mathcal{K}(\psi)$, by arguing as above we can find a linearly ordered subset of $\mathcal{K}(\psi)$, call it $\mathcal{K}$, that contains $f$. Then we can find a maximal linearly ordered subset $S$ of $\mathscr{K}(\psi)$ that contains $\mathcal{H}$. Let $g=\wedge \mathcal{G}$ be the pointwise infimum of all functions in $\mathcal{G}$. Then $f \geq$ $\wedge \mathfrak{H C} \geq \wedge \mathcal{G}=g$. From Theorem 4 we know that $g \in$ $\mathcal{K}(\psi)$. Also, $g$ is minimal in $(\mathcal{K}(\psi), \leq)$ because $\mathcal{G}$ is maximal. Hence, $g \in \mathbb{B}(\psi)$ and $f \geq g$.

Q.E.D.

\section{Proof of Theorem 5}

a) Let $\mathcal{K}$ and $B$ denote the kernel and basis of $\Psi$. If $A$ $\in \mathfrak{K}$, then from Theorems $4 \mathrm{a}$ and $\mathrm{A} 1$ there is $M \in \mathbb{B}$ such that $A \supseteq M$. Thus, $A \in \cup_{M \in B} \mathcal{E}(M)$. Hence, $\mathfrak{K} \subseteq$ 
$\cup_{M \in \mathbb{B}} \mathcal{E}(M) \subseteq \cup_{F \in \mathscr{K}} \mathcal{E}(F)$; also (25) is equivalent to $\mathcal{K}=\cup_{F \in \mathcal{K}} \&(F)$. Therefore, $\pi=\cup_{M \in \mathbb{B}} \&(M)$, and the proof of (38) is complete because of Proposition $1 b$.

b) From part a), $\Psi(X)=\cup_{M \in \mathbb{B}} X \ominus M$. Applying Theorem 5a to $\Psi^{d}$ we obtain

$$
\begin{aligned}
\Psi(X) & =\left[\Psi^{d}\left(X^{c}\right)\right]^{c}=\left[\bigcup_{N \in B\left(\Psi^{d}\right)} X^{c} \ominus N\right]^{c} \\
& =\bigcap_{N \in B\left(\Psi^{d}\right)} X \oplus \check{N},
\end{aligned}
$$

because $\left(X^{c} \ominus N\right)^{c}=X \oplus \check{N}$. Thus the proof of (39) is complete.

Q.E.D.

\section{Proof of Theorem 6}

a) Let $\mathscr{K}$ and $B$ denote the kernel and basis of $\psi$. If $f$ $\in \mathfrak{K}$, then from Theorems $4 \mathrm{~b}$ and $\mathrm{Al}$ there is $g \in \mathcal{B}$ such that $f \geq g$. Thus, $f \in \bigcup_{g \in \mathcal{B}} \varepsilon(g)$. Hence, $\mathfrak{K} \subseteq$ $\bigcup_{g \in \mathbb{B}} \mathcal{E}(g) \subseteq \cup_{h \in \mathcal{K}} \mathcal{E}(h)$; also, (34) is equivalent to $\mathcal{K}$ $=\cup_{h \in \mathcal{K}} \varepsilon(h)$. Therefore, $K=\cup_{g \in \Theta} \delta(g)$; then Proposition $4 \mathrm{~b}$ completes the proof of (40).

b) From part a), $\psi(f)=\vee_{g \in \Theta} f \ominus g$. Applying Theorem 6 a to $\psi^{d}$ yields

$$
\begin{aligned}
\psi(f) & =-\psi^{d}(-f)=-\underbrace{\vee}_{h \in \mathscr{B}\left(\psi^{d}\right)}(-f) \ominus h \\
& =\bigwedge_{h \in \mathscr{B}\left(\psi^{d}\right)} f \oplus \tilde{h} .
\end{aligned}
$$

Thus the proof of (41) is complete.

c) $\Phi$ is defined on the class $S$ of the threshold sets of all $f \in \mathfrak{F}$; thus $S$ is closed under translation and any intersection. From Lemma A1, $\Phi$ is TI increasing and upper semicontinuous. Hence, from (21) and Theorem 5a (applied to $\Phi)$ we obtain $T_{a}[\phi(f)]=\Phi\left[T_{a}(f)\right]=$ $\cup_{M \in B(\Phi)}\left[T_{a}(f)\right] \ominus M=\bigcup_{M \in ß(\Phi)} T_{a}(f \ominus M), \forall a \epsilon$ $\boldsymbol{V}$. Then property (9) completes the proof of (42).

d) The sup-of-erosions representation for $\phi$ was proven in c). Applying Theorem $5 b$ to $\Phi$ yields $T_{a}[\phi(f)]=$ $\Phi\left[T_{a}(f)\right]=\bigcap_{\left.N \in B_{(\Phi)}\right)}\left[T_{a}(f)\right] \oplus \bar{N}=\bigcap_{N \in \mathbb{B}\left(\Phi^{d}\right)} T_{a}(f$ $(\bar{N}), \forall a \in V$. Then property (8) completes the proof of (43).

Q.E.D.

\section{Proof of Proposition 6:}

Let $W=A \oplus \check{A}$ and $\cap$ be the basis of the closing by

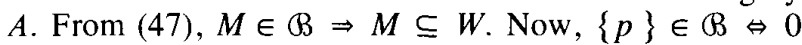
$\in\{p\} \bigcirc A \Leftrightarrow p=0$. Assume $n \geq 2$. If there are sets $\{a, b\}$ in $B$, then $a, b \in W \backslash\{0\}$. Then $\{a, b\} \bigcirc A=$ $(A+a \cup A+b) \ominus A$. Hence (assume $a<b), 0 \in\{a$, b) $A \Leftrightarrow A \subseteq(A+a \cup A+b)=\{x: a+c \leq x \leq$ $a+c+n-1$, or $b+c \leq x \leq b+c+n-1\}$. This is true iff $a<0<b$ and $b-a \leq n$. For each $k=b-$ $a, 2 \leq k \leq n$, there are $k-1$ sets $\{a, b\}$ in $\mathbb{B}$ since 1 $\leq b \leq k-1$. Hence there are totally $n(n-1) / 2$ such sets $\{a, b\}$ in $B$. Finally, for all $M \in B,|M| \leq 2$. To see this, observe that $0 \in M \bullet A \Leftrightarrow A \subseteq U_{x \in M} A+x$. If $0 \notin M$ and $M \in \mathbb{B}$, then there must exist $a, b \in M$ with $a$ $<0<b$; choose the largest such $a$ and the smallest such $b$. If there is an additional point $e \in M$, then either $b<e$ or $e<a$. If $b<e$, then the points of $A+e$ needed in the union $\cup_{x \in M} A+x$ to cover $A$ have already been contributed by $A+b$; similarly, we exclude the case $e<a$. Thus, $B$ consists exactly of $n(n-1) / 2$ two-point sets and the set $\{0\}$.

Q.E.D.

\section{ACKNOWLEDGMENT}

The guidance, insights, and encouragement provided by Prof. R. W. Schafer during the Ph.D. dissertation research reported here were extremely helpful and are gratefully acknowledged. Thanks also go to two anonymous reviewers and to Dr. C. Ronse at Philips for their helpful and detailed comments.

\section{REFERENCES}

[1] G. Matheron, Random Sets and Integral Geometry. New York: Wiley, 1975.

[2] J. Serra, Image Analysis and Mathematical Morphology. New York: Academic, 1982.

[3] H. Hadwiger, Vorlesungen über Inhalt, Oberfläche, und Isoperimetrie. Berlin: Springer-Verlag, 1957.

[4] S. R. Stcrnberg. "Cellular computers and biomedical image process," in Biomedical Images and Computers, J. Sklansky and J. C. Bisconte, Eds. Berlin: Springer-Verlag, 1982.

[5] — , "Grayscale morphology," Comput. Vision, Graphics, Image Processing, vol. 35, pp. 333-355, 1986.

[6] Y. Nakagawa and A. Rosenfeld, "A note on the use of local min and max operations in digital picture processing," IEEE Trans. Syst., Man, Cybern., vol. SMC-8, Aug. 1978.

[7] C. Lantuejoul and J. Serra, "M-Filters," in Proc. IEEE Int. Conf. Acoust., Speech, Signal Processing, Paris, France, May 1982, pp. 2063-2066.

[8] T. R. Crimmins and W. R. Brown, "Image algebra and automatic shape recognition," IEEE Trans. Aerosp. Electron. Syst., vol. AES21, pp. 60-69, Jan. 1985.

[9] P. Maragos, "A unified theory of translation-invariant systems with applications to morphological analysis and coding of images," Ph.D. dissertation, School Elec. Eng., Georgia Inst. Technol., Atlanta, July 1985; also Tech. Rep. DSPL-85-1.

[10] _ , "Tutorial on advances in morphological image processing and analysis," Opt. Eng., July 1987; also in Proc. SPIE 707, 1986.

[11] P. Maragos and R. W. Schafer, "A unification of linear, median, order-statistics, and morphological filters under mathematical morphology," in Proc. IEEE Int. Conf. Acoust., Speech, Signal Processing, Tampa, FL, Mar. 1985, pp. 34.8.1-34.8.4.

[12] - , "Morphological Filters-Part I: Their set-theoretic analysis and relations to linear shift-invariant filters," IEEE Trans. Acoust., Speech, Signal Processing, vol. ASSP-35, Aug. 1987.

[13] 1 . "Morphological filters-Part II: Their relations to median, order-statistic, and stack filters," IEEE Trans. Acoust., Speech, Signal Processing, vol. ASSP-35, Aug. 1987

[14] R. M. Haralick, S. R. Sternberg, and X. Zhuang, "Image analysis using mathematical morphology," IEEE Trans. Pattern Anal. Machine Intell., vol. PAMI-9, pp. 523-550, July 1987.

[15] R. M. Haralick, C. Lin, J. S. J. Lee, and X. Zhuang, "Multiresolution morphology," in Proc. Ist ICCV, London, 1987.

[16] K. Preston, Jr., M. J. B. Duff, S. Levialdi, P. E. Norgren, and J-I. Toriwaki, "Basics of cellular logic with some applications in medical image processing," Proc. IEEE, vol. 67, pp. 826-856, May 1979.

[17] V. Goetcherian, "From binary to grey tone image processing using fuzzy logic concepts,' Pattern Recognition, vol. 12, pp. 7-15, 1980.

[18] A. Rosenfeld and A. C. Kak, Digital Picture Processing, vols. 1 \& 2. New York: Academic, 1982.

[19] T. Esselman and J. G. Verly, "Applications of mathematical morphology to range imagery," MIT Lincoln Lab, Tech. Rep. 797, Dec. 1987.

[20] T. R. Hsing, Ed., Proc. SPIE (Visual Communications and Image Processing II), vol. 845, 1987.

[21] J. C. Klein and J. Serra, "The texture analyzer," J. Microscopy, vol. 95 , pt. 2 , pp. $349-356$, Apr. 1972. 
[22] M. J. B. Duff, D. M. Watson, T. J. Fountain, and G. K. Shaw, "A cellular logic array for image processing," Pattern Recogn., vol. 5, pp. 229-247, 1973.

[23] R. M. Lougheed, D. L. McCubbrey, and S. R. Stemberg, "Cyto computers: Architectures for parallel image processing," in Proc. Workshop Picture Data Descr. Manag., Pacific Grove, CA, 1980.

[24] Proc. IEEE Workshop CAPAIDM, Miami, FL, Nov. 1985.

[25] R. G. Harber, S. C. Bass, and G. W. Neudeck, "VLSI implementation of a fast rank order filtering algorithm," in Proc. IEEE Int. Conf. Acoust., Speech, Signal Processing, Tampa, FL, Mar. 1985, pp. 1396-1399.

[26] E. Ochoa, J. P. Allebach, and D. W. Sweeney, "Optical median filtering by threshold decomposition," Appl. Opt., vol. 26, pp. 252260, Jan. 1987

[27] K. S. O'Neill and W. T. Rhodes, “Morphological transformations by hybrid optical-electronic methods," in Proc. SPIE (Hybrid Image Processing), D. Casasent, Ed., vol. 638, pp. 41-44, 1986.

[28] J. M. Hereford and W. T. Rhodes, "Nonlinear optical image filtering by time-sequential threshold decomposition," Opt. Eng., May 1988.

[29] P. E. Miller, "An investigation of Boolean image neighborhood transformations,'” Ph.D. dissertation, Ohio State Univ., 1978.

[30] —, "Development of a mathematical structure for image processing,' Perkin-Elmer Opt. Division, Tcch. Rcp., 1983.

[31] G. X. Ritter, J. L. Davidson, and J. N. Wilson, "Beyond mathematical morphology," in Proc. SPIE (Visual Commun. Image Processing $/ 1$ ), vol. 845, pp. 260-269, 1987.

[32] G. X. Ritter and P. D. Gader, "Image algebra techniques for parallel image processing," J. Parallel Distrib. Comput., vol. 4, pp. 7-44, 1987.

[33] G. X. Ritter and J. N. Wilson, "Image algebra in a nutshell," in Proc. 1st ICCV, London, June 1987, pp. 641-645.

[34] E. R. Dougherty and C. R. Giardina, "Image algebra-induced operators and induced subalgebras," in Proc. SPIE (Visual Commun. Image Proces. II), vol. 845, 1987, pp. 270-275.

[35] " "A digital version of the Matheron representation theorem for increasing $\tau$-mappings in terms of a basis for the kernel," in Proc. IEEE Conf. Comput. Vision, Pattern Recogn., Miami, FL, June 1986, pp. 534-536.

[36] A. Kaufmann, Introduction to the Theory of Fuzzy Subsets. New York: Academic, 1975.

[37] J. P. Fitch, E. J. Coyle, and N. C. Gallagher, "Median filtering by threshold decomposition,' IEEE Trans. Acoust., Speech, Signal Processing, vol. ASSP-32, pp. 1183-1188, Dec. 1984.

[38] P. D. Wendt, E. J. Coyle, and N. C. Gallagher, "Stack filters,"
IEEE Trans. Acoust., Speech, Signal Processing, vol. ASSP-34, pp. 898-911, Aug. 1986

[39] W. Köhler, Gestalt Psychology. New York: Liveright, 1970.

[40] A. N. Kolmogoroff and S. V. Fomin, Introductory Real Analysis. New York: Dover, 1975

[41] R. L. Stevenson and G. R. Arce, "Morphological filters: Statistics and further syntactic properties," IEEE Trans. Circuits Syst., vol. CAS-34, pp. 1292-1305, Nov. 1987.

[42] T. S. Huang, Ed., Two-Dimensional Digital Signal Processing II Transforms and Median Filters. New York: Springer-Verlag, 1981.

[43] G. R. Arce, N. C. Gallagher, and T. A. Nodes, "Median filters: Theory for one- and two-dimensional filters," in Advances in Computer Vision and lmage Processing, vol. 2, T. S. Huang, Ed. JAI Press, 1986

[44] T. A. Nodes and N. C. Gallagher, Jr., "Median filters: Some modifications and their properties," IEEE Trans. Acoust., Speech, Signal Processing, vol. ASSP-30, pp. 739-746, Oct. 1982

[45] A. C. Bovic, T. S. Huang, and D. C. Munson, Jr., "A generalization of median filtering using linear combinations of order statistics," IEEE Trans. Acoust., Speech, Signal Processing, vol. ASSP-31, pp. $1342-$ 1349, Dec. 1983

[46] H. J. A. M. Heijmans and C. Ronse, "The algebraic basis of mathematical morphology. Part I: Dilations and erosions," C. W. I., Amsterdam, The Netherlands, Rep. AM-R8807, June 1988.

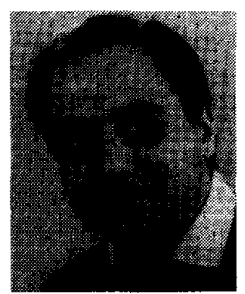

Petros Maragos (S'81-M'85) was born in $\mathrm{Ka}$ lymnos, Greece, on November 4, 1957. He received the Diploma degrec in elcetrical engineering from the National Technical University of Athens, Greece, in 1980, and the M.S.E.E. and Ph.D. degrees from the Georgia Institute of Technology, Atlanta, in 1982 and 1985, respectively.

From 1980 to 1985 he was a Research Assistant at the Digital Signal Processing Lab of the Electrical Engineering School at Georgia Tech. Since August 1985 he has been an Assistant Professor of Electrical Engineering in the Division of Applied Sciences at Harvard University, Cambridge, MA. His current research interests include signal and image processing, computer vision, and pattern recognition.

Dr. Maragos received a National Science Foundation Presidential Young Investigator Award in 1987. 